\title{
The botany of the Cunene-Zambezi Expedition with notes on Hugo Baum (1867-1950)
}

\author{
E. FIGUEIREDO**, M. SOARES**, G. SEIBERT***, G.F. SMITH*and R.B. FADEN*•
}

Keywords: Angola, Baum, collectors, flora

\section{ABSTRACT}

\begin{abstract}
A record and discussion of the Cunene-Zambezi Expedition that took place in Angola from 1899 to 1900 is provided Although the main aim of the expedition was to evaluate the economic potential of southern Angola. it also resulted in significant botanical collections made by Hugo Baum (1867-1950), many of which serve as holotypes of names of plants from the region, and beyond. The itinerary is supplemented by a map illustrating the route followed by the expedition, and locality names are clarified and updated. A full list of the type specimens of flowering plants is provided, with their nomenclature updated and an indication of where known duplicates are housed.
\end{abstract}

\section{CONTENTS}

Abstract....................... 185

Introduction ................... 185

Material and methods ............... 187

Historical overview and itinerary of the expedi-

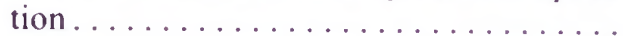

List of Baum type collections given in Warburg

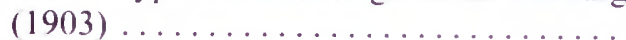

Acknowledgements. . . . . . . . . . . . . . . 201

References...................... 201

Index to plant names. . . . . . . . . . . . . 203

Index of Baum collection numbers cited and taxon under which they are cited in this paper ... 206

Appendix 1..................... 208

\section{INTROLUCTION}

The collector Hugo Baum (1867-1950) participated in the Cunene-Zambezi Expedition, which took place in Angola from 1899 to 1900 , led by the Dutchman, Pieter van der Kellen. In addition to botanical and zoological collections, ethnological observations were also recorded. All of these were described in German by Warburg (1903) in the now very rare book Kunene-Sambesi Expedition. H. Baum, which can only be found in highly specialized libraries or in the collections of rare book antiquarians. The book includes a folded map (Figure 1) that illustrates the course of the expedition, which left Moçâmedes (Namibe) on 11 August 1899 in an easterly direction, through the actual provinces of Cunene and Cuando-Cubango, reaching

\footnotetext{
* H.G.W.J. Schweickerdt Herharium, Iepartment of Botany, University of Pretoria. (N)(2 Pretoria. estrelafigueiredora hotmail.com.

* Departamento de Botanica. Universidade de Coimbra. 3(k)1-455 Coimbra. Portugal. miguel.vonberg(a gmail.com

*** Centro de Estudos Africanos (CEA)/SCTE. Lisbon. Portugal. mailsciberta yahox.com

- Biosystematics and Biodiversity Collections, South African National Biodiversity Institute. Private Bag X|0). (N)(0) Pretoria/Acocks Chair. H.G.W.J. Schweickerde Herbarium, Department of Botany. University of Pretoria. (0)(0)2 Pretoria. smithg(a sanbi.ory.

- Department of Botany, MRC 166. National Muscum of Natural History. Smithsonian Institution. P.(). Box 370)12, Washington I) (2)(0)137012, USA. fadenr a si.edu

+ Corresponding author. Email address: estrelafigueiredo a hotmail.com. MS. received: $20(19-0) 1-14$
}

the Cuando River in March 1900. After turning around, the expedition finally arrived back at Moçâmedes on 26 June 1900, more than 10 months later.

The expedition resulted in over 1000 plant collections, many of which are types. In Warburg (1903), 281 taxa of flowering plants new to science are described based on these collections, with 310 numbers referred to as types. To these must be added an unknown number of taxa that were published later. In the Aluka database of types of African plant names (Aluka 2008. accessed in January 2008), there are 1209 images of specimens collected by Baum. This figure includes duplicates of the same collection number. In fact, the Baum collections provided a series of duplicates distributed among European herbaria. This is fortunate because the holotypes of the taxa published in Warburg (1903) were deposited in the Herbarium of Berlin (B) and many of these specimens were destroyed in a fire during the Second World War. For that reason the Aluka database presently hosts only 48 images of Baum specimens from B. Duplicates have been recorded in various herbaria, namely $\mathrm{B}, \mathrm{BM}$, BR, COI, E, G, K, M, NY, P, S, W and Z (Holmgren, et al. 1990), which include several herbaria that were not listed as depositories of Baum collections in Lanjouw \& Stafleu (1954). However, for some taxa, the holotype destroyed at B was apparently unique.

In terms of type material the Baum collection is one of the most important among those that originated in Angola, and is only surpassed by those of F. Welwitsch and J. Gossweiler. In addition to their type status, they provide information on type locality. This is very important in the case of rare plants, particularly those that were collected only once. Information on the exact collecting localities helps researchers find rare plants in the field. which in turn facilitates the eventual proposal of specific conservation measures. In the case of the Baum collection, pinpointing the collecting localities is often difficult for several reasons: 1, localities recorded on the labels are often not consistent with duplicates of the same collection, or localities given in the book; 2 , the changes of geographical place names that took place in Angola over the last century: and 3, the original spelling of the localities that does not match the more recent spelling used in maps and gazetteers. 

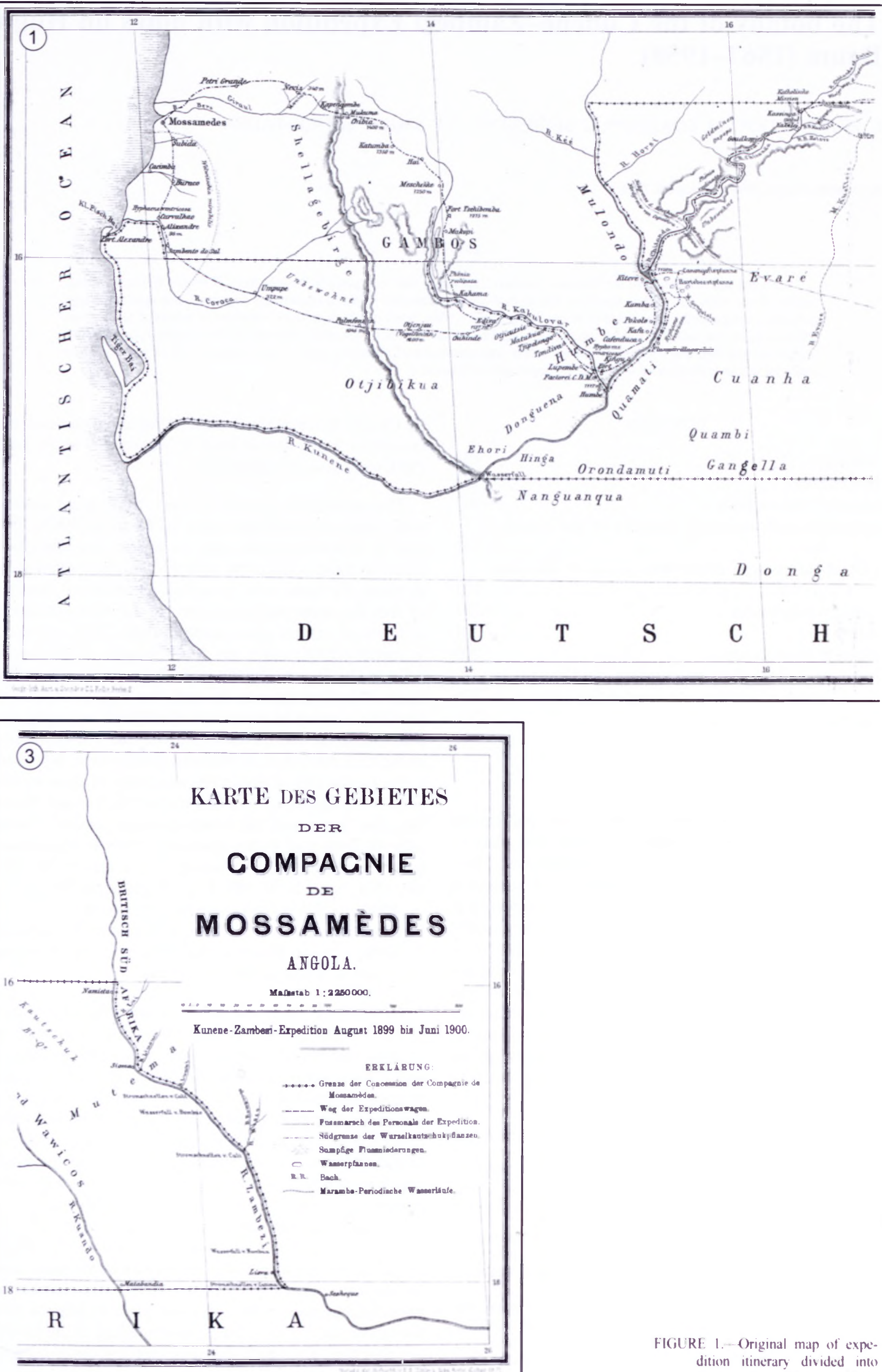


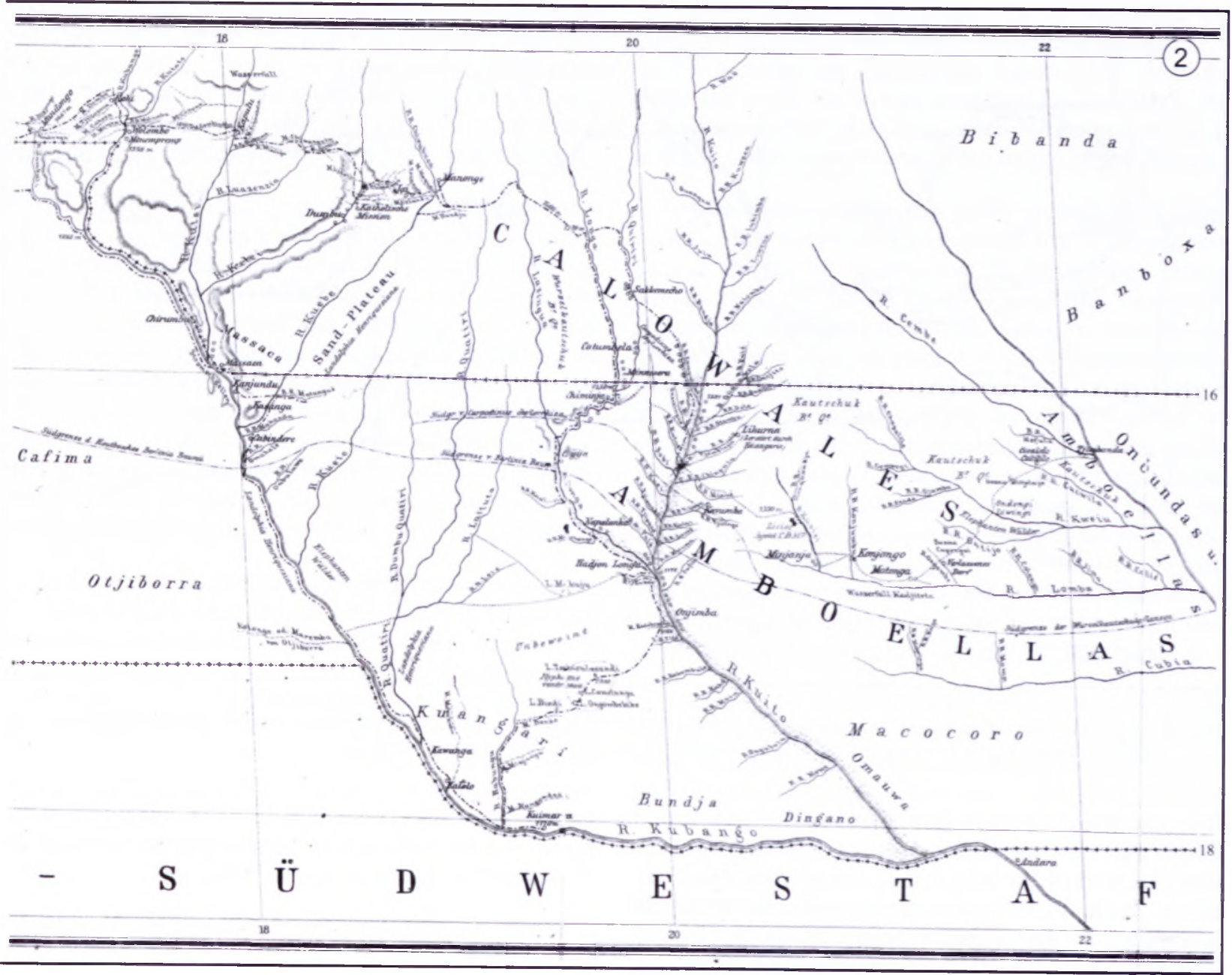

To facilitate the work of taxonomists and systematists studying the flora of southern Africa, the Baum collections and expedition itinerary were analysed. In the present work we include an historical overview of the expedition and an analysis of the flowering plant type material cited in the book (Warburg 1903). Based on the original map, a new map has been produced, which is complemented by a list of old locality names, as cited in Warburg (1903), and the corresponding current names and, whenever possible, geographical co-ordinates.

\section{MATERIAL AND METHODS}

The information on type collections was obtained from several sources. Initially, the information on types cited in Warburg (1903) was databased, using Figueiredo $\&$ Smith (2008) to update the nomenclature. The location of duplicates was determined mostly using internet resources. In this regard, the Aluka database (www.aluka. org) was particularly useful. Several herbaria online, such as $\mathrm{K}$ (www.kew.org herbcat), B (www.bgbm.org) and Z (http:/www.zuerich-herbarien.unizh.ch/) also provided much information. Duplicates cited in the literature but not seen (either in herbaria or as images) were included in the database. Information from COI was obtained from a card catalogue. The existence of B types was verified using the resources mentioned above and a list of families including extant collections at B (Hiepko 1987). It was noted that the localities recorded on the labels are often not consistent with duplicates of the same collec- tion. Furthermore, they often do not match exactly the localities given in the book. As a compromise the collecting localities given in Warburg (1903) are used.

The types are listed in alphabetical order by family, genus and species, in their currently accepted taxonomy (Figueiredo \& Smith 2008). An index to all the scientific names as well as a sequential index of type numbers referring to their name in this list, is also presented.

A list of several geographical names, cited in Warburg (1903) as collecting localities, as well as those shown on the map, was compiled. The location of the names was determined using maps and gazetteers. An annotated copy of the Warburg (1903) map, produced at LISC, was examined. A new map (Figure 2) showing the itinerary and updated locality names was produced based on the information compiled.

\section{HISTORICAL OVERVIEW AND ITINER ARY OF THE EXPEDITION}

Hugo Baum was born on 17 January 1867 in Forst Niederlehmsitz, the son of a railwayman. He was raised in Guben on the river Neisse (then in Brandenburg, Germany: now Polish and called Gubin) in a forested area where he developed an interest in plants and insects. After concluding his school education in Guben, he started his professional training as a gardener in Nettkow (Silesia; then German now Polish) and later attended a 


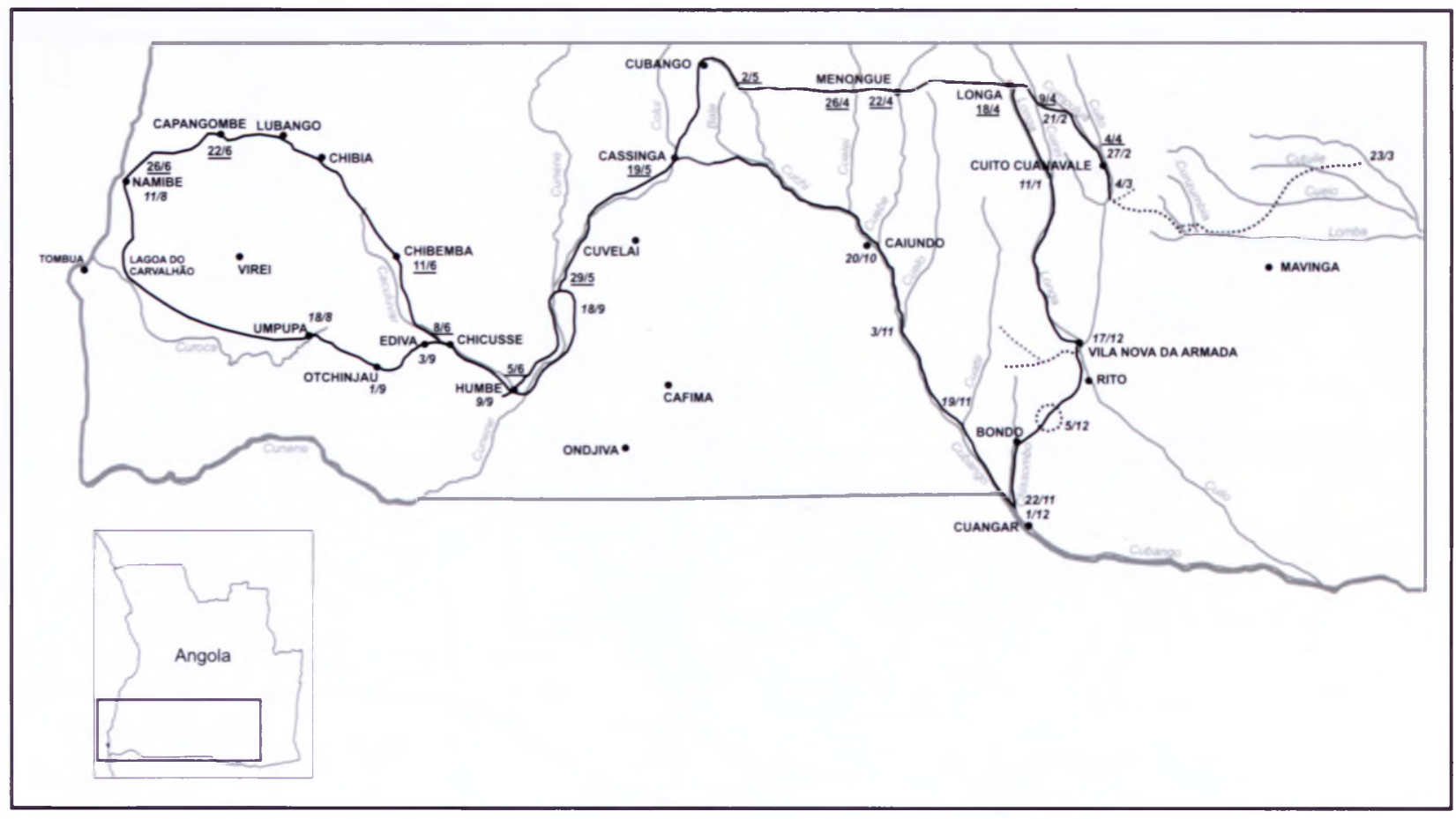

FIGURE 2.- Actual map of expedition itinerary showing route taken by ox-wagons (continuous line), routes travelled on foot (dotted lines), and dates when some localities were reached (in italics for journey out, underlined for return journey).

two-year study course in pomology in Proskau (Silesia). Following his compulsory military service in Magdeburg, he worked for ten years at the old Botanical Garden in Berlin. In 1899, thanks to his extensive botanical knowledge, he was invited to participate in the CuneneZambezi Expedition. After his return from Angola, in 1901, Baum was employed as master gardener by the Botanical Garden of the University of Rostock. In the same year he married Auguste Tank. For the following few years Baum busied himself evaluating the CuneneZambezi Expedition and in 1903 the expedition report was published. Baum undertook another botanical expedition to Mexico, from January to May 1925. In 1926 he was promoted to chief garden inspector (Gartenoberinspector) of the University of Rostock, and in 1933, at the age of 66 , he retired. On 15 April 1950, shortly after his 83 rd birthday, Baum died impoverished. His wife passed away the following year. Altogether 71 taxa, including two genera, namely Baumiella (a mushroom) and Baumia (belonging to the Scrophulariaceae), have been named after Baum (Mansfeld 1983; Heintze 2007).

The Cunene-Zambezi Expedition was carried out at the request of the Berlin-based Colonial-Economic Committee together with the Compagnie de Moçâmedes (Paris) and the South West Africa Company (London). Its objective was to investigate the economic potential of the south of Angola. The expedition was the result of the interest of German Southwest Africa (now Namibia) in the planned train connection between Porto Alexandre (presently Tombua) in Angola and the then independent Zuid-Afrikaanse Republiek, later the Transvaal province of South Africa. At the time, there was a lack of knowledge of wild-growing cash crops and local African cultures in the area. In his expedition report Hugo Baum did not only describe the flora, but also provided information on the geography, geology, climate, the local people encountered on the expedition, and some of their cus- toms. The leader of the expedition was the Dutchman, Pieter van der Kellen, who was the representative of the Compagnie de Moçâmedes in Ediva.

On 11 August 1899, the expedition left Moçâmedes (presently Namibe) with three ox-wagons for the rivers Kunene, Kubango, Kuito, up to the Cuando, and on 26 June 1900 it returned to the place from which it departed (Figures 1, 2; refer to Appendix 1 for actual name and co-ordinates of localities, which are mentioned in this text with the original spelling). At the time Moçâmedes was a town of 1500 inhabitants with a local economy dominated by the trade in dried fish.

The most common acacia species found between Ediva and Humbe was Acacia verrucifera Harms [= Acucia kirkii Oliv. subsp. kirkii var. kirkii] that provided the best transparent gum (gummi arabica) of great bonding strength. The distribution area of this acacia extended from the Chela Mountains to the Kunene River, and from the Chitanda and the Kubango Rivers downwards to the territory of the Kuangari tribe. Near Chihinde, Baum collected Strophamthus amboensis (Schinz) Engl. \& Pax of which only a few plants were found. At the same location he found for the first time a plant that was referred to as Gardenia thunbergia Thunb. [of which the collected specimen is Gardenia volkensii K.Schum. subsp. spathulifolia (Stapf \& Hutch.) Verdc.], which was called stumpdorn. the German version of the Afrikaans stompdoring, a name recorded by Smith (1966). and mulavi by the local Africans. This tree appeared frequently along the Kunene. Chitanda and Kubango Rivers, but also along the Longa and Kuito Rivers and grew predominantly in the low grounds of the rivers or on the river islands.

The expedition arrived at Ediva on 3 September and there they met the leader Van der Kellen and two Boers 
and two Africans with horses, who had been hired by the Dutchman at Humpata as hunters to provide game meat for the expedition. Van der Kellen's brother Emil managed a large farm in Ediva. It was owned by the Compagnie de Moçâmedes, and cultivated various food and cash crops, and carried between 800 and 1000 head of cattle.

After Ediva, the first place reached by the expedition on their way to Humbe was called Otjikutsie. Humbe used to have a trading post for cattle. However, it had lost importance since the outbreak of the cattle plague. The local population in Humbe cultivated sorghum, pennisetum, groundnuts, groundpeas and to a lesser extent beans, locally called macundi. From Humbe the expedition travelled on the same day to the Kunene River. On 12 September they crossed the Kunene with the support of the people of Humbe, of whom some 40 men had joined the expedition as porters. On the left bank of the Kunene the expedition headed upstream to the mouth of the Chitanda River.

At the Chitanda, the houtbosch, Berlinia haumii Harms [= Julbernardia paniculata (Benth.) Troupin], which they considered the most common tree in southern Angola, was noticed for the first time. The local population used the bark of this tree for the production of boats. In Goudkopje, on the banks of the Chitanda, a place also locally called Ompopo, the expedition spent several days searching for alluvial gold in the river; they did find a small quantity. In early October the expedition reached Kassinga. also on the banks of the Chitanda. From there, they went on to the Nambali stream which flows into the Kubango River.

On 8 October the explorers reached the Kubango at Linghonung, the first village they came across since Kassinga. Following the Kubango, on 15 October the expedition came to the village of Chirumbo, located on a river island to protect the population against assaults by the Kuinghama tribe (Kwanhama?) from Ovamboland. On 16 October they crossed the Kubango for the first time beween the Kutsi River and Massaca, a fortified village inhabited by some 500 people. From Massaca the explorers travelled the Kubango downstream to a village called Kanjundu, where they left the Kubango to proceed to the Kuebe River, which they followed downstream to its confluence with the Kubango. Then they continued along the Kubango to the mouth of the Quatiri River. Downstream from the mouth of the Quatiri, the expedition passed through the village of Kavanga on the Kubango that was inhabited by the Kangella tribe. From there, the explorers followed the Kubango downstream along the border with German Southwest Africa (presently Namibia) to Imbala, the principal village of the Kuangaris. On 27 November, the expedition left Imbala accompanied by a dozen Kuangari guides following the Kubango upstream to the confluence with the Habungu and then along this stream further upstream.

From the Habungu the journey continued to the Bondo, a small stream that contained little water even during the rainy season, which they followed for only a small distance. From there the expedition went to the lake-like pan called Ungombekike from where some of the Kuangari guides returned home. After passing through two other pans the group reached the Kuito River. Moving upstream along the Kuito, after a few days the group reached the mouth of the stream Jonkoa and from there. the nearby mouth of the Longa. On 26 December, the explorers arrived at the village Napalanka, situated in a valley between the Longa and inaccessible swamps. After following the Longa upstream for four days, the expedition arrived at a place called Minnesera, which is situated in the swampy area of the mouth of the Quiriri.

On 16 January 1900, the group crossed the Longa, of which the banks were so swampy that 80 oxen were needed to pull the wagons out of the swamp. Downstream from the mouth of the Quiriri the expedition erected a camp where they were visited by the chief of Minnesera and 30 of his people. A few kilometres downstream from Minnesera, the Longa was crossed again with a float. On 31 January the expedition reached the same location at the Longa where they had been on the 12th. Due to a lack of food supplies for the expedition, following the local population's refusal to barter for food supplies, sweet potatoes were simply taken in sacks from nearby fields to feed the carriers and the horses. The shortage of maize rations had weakened the horses, which forced the expedition to reduce the hunting parties, which, in turn, resulted in a lack of game meat.

Subsequently the expedition reached a ford at the Longa, which rubber dealers used as passage-way. At that place, several residing dealers exchanged root rubber, $\mathrm{Car}$ podimus chylorrhiza K.Schum. [= Landolphia thollonii Dewèvre] for commodities. Two-wheeled carriages were also sent to collect the rubber in the surrounding region. The dealers kept considerable rubber stocks tied up in bundles of 40 finger-strong rubber strips (see below), which as an exchange unit were called manga. The weight of one manga was about one kilogram. The expedition managed to purchase eleven sacks of maize from a local Portuguese man. That became their only foodstuff and had to be rationed. Due to the food shortage, 17 of the porters were sent back to Humbe and Ediva.

One of the ox-wagons left the Longa for the Quiriri, while the two others remained at the Longa to trade. The expedition avoided contact with the Portuguese traders at the Quiriri, because '...the Portuguese in the colonies were often deported persons...' (Warburg 1903: 93). However. they associated with the Swedish trader Swanström who sent them freshly baked bread. Later the two ox-wagons that were left at the Longa rejoined the expedition at the Quiriri, where in a few days, 300 mangas would be exchanged for cotton cloth. From the Quiriri downstream the inhabitants of the many villages were busy with the preparation of rubber. In a village at the Quiriri called Sakkemecho, the expedition exchanged consumer goods and cattle for 297 mangas on credit that would be collected after a few months during the return journey.

In the rubber-producing area, rubber completely replaced money as local currency. Root rubber was predominantly found on large grass fields on sandy soils, called chanas (samnas), which were surrounded by forests. The plant did not grow near the streams and rivers, but on the waterless chanas between the streams. Formerly, the root rubber plant had occurred commonly at the Kutsi and the Kubango Rivers at Massaca. where it had now become completely extinct. However, the plant was said to exist in the territory of the Kuinghama tribe and occurred above the mouth of the Luassingua, reach- 
ing the Longa, Quiriri, Kampuluve and Kuito Rivers. Its range went beyond the latter and extended even as far as the Cuando. After having been dug out with short hoes, the roots, tied up into stacks of $\pm 2 \mathrm{~m}$ tall, were watered regularly to soften the bark so that it later peeled off easily. Thereafter, the bales were again dried in the sun and the single roots were cut into 30 to $40 \mathrm{~cm}$ long pieces that were beaten on a board to remove the bark from the wood. The pieces of bark were then worked with a wooden hammer until they took the form of a cake. This cake was boiled and again beaten with clubs. Finally the cake was cut into square pieces, which were placed in boiling water and, while still hot, were shaped into cylindrical, finger- to thumb-thick strips, about the length of a span of the hand $( \pm 22 \mathrm{~cm})$ called matali, of which 40 pieces constituted one manga. There were a total of eleven traders in the entire rubber area, of whom nine were Portuguese, one was an Englishman and one a Swede.

The expedition continued the journey from Sakkemecho, crossing a waterless and hilly area between the Quiriri stream and the Kampuluve, which ran into the Kuito River. The expedition reached the Kampuluve at its source in a swampy valley that extended for 25 to 30 $\mathrm{km}$ up to the mouth of the stream. They arrived there on 27 February 1900 and reached the Kuito River for the second time. At that point the Kuito was at most $150 \mathrm{~m}$ wide, but with very deep and fast-flowing water. On 14 March, the expedition crossed the Kuito with canoes and on the same day reached a stream called Chinpulu on foot. Due to the lack of grass for the oxen at the Kuito the wagons and oxen had been sent back with two people to the Onschingue River where the others were expected to arrive at the end of March. From the Chinpulu River the men reached a small stream called Miané that they followed up to its source. Then the march continued through waterless hills to the Kuisi stream in the Zambezi area. The expedition followed the Kuisi downstream, then crossed it and after several hours reached the village Konjongo at the Konjumbu, a small stream.

By the time the expedition reached Konjongo, Baum was weakened by fever and stomach pains and he decided to return with two porters to the Kuito River, which they crossed to the right bank. From there the group went to the Onschingue where on 1 April they met part of the expedition that had remained at the Cuando. On 4 April, the expedition initiated the return journey along the previous route, upstream along the Kuito and Kampuluve Rivers up to the Sakkemecho village, where they delivered to the local inhabitants part of the rubber they owed them from the previous transaction.

From the Sakkemecho village, the men followed the Quiriri upstream and then crossed the sandy hills to the Longa. From the Longa the expedition travelled to the Lazingua, and then westwards passing the Quatiri to the Bundju stream, where they remained for some time to allow the oxen some rest. The journey continued to the Kuebe and the Kulei, a tributary of the Kutsi. The passage across the Kutsi, which was rather deep, took three days. From there the journey went on to the Kubango. The crossing of the Kubango with more than a hundred head of cattle and the wagons that had to be dissembled to be ferried across the river, took another five days. The group then followed the Cubango upstream and turned westward behind Kohi.

On 19 May, the expedition arrived at the Chitanga River and shortly afterwards at nearby Kassinga. After a half-day stay in Kassinga, the expedition followed the Chitanga downstream to Goudkopje. The Chitanga area was rich in game. On 29 May the expedition reached the Kunene at Kiteve. The local population provided eight large boats to help the expedition cross the Kunene within two days. From Kiteve the expedition followed the Kunene downstream. On 5 June the men arrived at Humbe where the porters from that place left the expedition after having been paid for their services. For the remaining journey to the coast the expedition recruited other local porters. On their way to Ediva at the Kaculovar River that was reached after two and a half days, they met the Dutchman Emil van der Kellen who had come by horse from Ediva when he had heard about the expedition's return.

At a place called Kahama, the expedition crossed the Kaculovar a second time and then followed it upstream. In the clay soils along the Kaculovar, Baum again frequently found Acacia kirkii Oliv., which was said to produce the best gum. Within two days the expedition crossed the passage through the Chela Mountains. After leaving the mountains, the men went along a stream called Tschikutt passing consecutively through Pietfontain [Pietfontein in modern Afrikaans], the dry Olefantsrivier [Olifantsrivier], the Bumbo and the Kitibe. At Monino village they had a longer rest before they walked the long and dry way to the Giraul River. From there the expedition travelled the last part to Moçâmedes, where they arrived on 26 June 1900.

During the ten-month journey, members of the expedition killed 175 antelopes, 3 hippos, 2 boars, and 3 crocodiles. Baum collected a total of 1016 plant collection numbers, of which more than 300 were later described as new taxa. More than 60) of the new species were named after Baum. Besides this haul, Baum caught more than 300 butterflies, prepared them and sent them to Europe.

With regard to the economic importance of southern Angola, Baum recommended the areas close to the coast at the small rivers Bero, Giraul, Monino, and Coroca for the establishment of plantations, particularly for the cultivation of cotton. In his opinion, the high plateau. predominantly constituted of heavy loamy or clay soils was suitable for the cultivation of maize, wheat, rye, oats, barley, and European vegetables, subtropical fruits such as citrus, and grapevines. According to Baum, there was much fertile land for colonization at the Cuando. The part of this area east of the Cuebe and north of the Lomba was characterized by a wealth of root rubber plants. He considered the region particularly rich in trees providing tanning agents, such as the mopane, Colophospermum mopane (J.Kirk ex Benth.) J.Léonard and the hourbosch. Julbernardia paniculata (Benth.) Troupin. The distribution area of the acacia that provided the best gum was located in the region of the Caculuvar and Kunene. Thanks to its large forests, southern Angola was considered very rich in timber; however, the cost of transport to the coast was very high. Although gold was panned in the Chitanda River at Goudkopje beneath 
Cassinga, the main value of southern Angola was that it was very suitable for livestock. In Baum's opinion the transport of cattle to the coast was easy, given the availability of water and pastures along the whole way. Finally, he emphasized the abundance of fish resources at Moçâmedes (Namibe), Tiger Bay, and particularly Porto Alexandre (Tombua), where salterns that produced salt for the preservation of fish were located.

\section{LIST OF BAUM TYPE COLLECTIONS GIVEN IN WARBURG (1903)}

All the holotypes (or syntypes) of the names published in Warburg (1903) were deposited at B. Information is available in the literature and on the internet concerning the families which survived the fire that consumed the herbarium during the Second World War (see www. bgbm.org). At the time of the bombardment, the staff of the herbarium was undertaking the task of transferring the type specimens to a safer location. Types belonging to certain families are therefore still extant at Berlin. Likewise, material on loan to other herbaria obviously also survived. The remaining material has been reported as lost. In the case of the Baum collections, out of the 311 type collection numbers of flowering plants cited in Warburg (1903), only 40 holotypes and four syntypes are confirmed to exist. There are 181 holotypes reported as destroyed, and 34 belonging to families for which type material apparently survived, could not be located in any of the databases cited above. In rare cases, types that were reported as destroyed in the literature were found to be extant as they are available in the Aluka database. This is the case for the holotype of Lobelia minuridentata Engl. \& Gilg, that had been reported as destroyed (Thulin 1984). This was probably due to the misplacement of the specimen as the locality data provided to Aluka wrongly assigns it to Namibia. It was noticed that several Baum collections have been attributed to the wrong country, and depending on the arrangement of herbaria, this may be a reason for the material not having been found in the past. For instance, Baum 894 (Cephalaria retroseta Engl. \& Gilg), is represented in Aluka (accessed in February 2008) by three duplicates: the K duplicate is attributed to South Africa, the BM duplicate to the Democratic Republic of Congo and only the duplicate in E is correctly assigned to Angola. In other cases it $w$ as found that types belonging to families that were reported as destroyed still exist. For example, in the Rubiaceae, at least four Baum types are extant.

Note: duplicate specimens in bold were accessible in Aluka (accessed in February 2008); duplicate specimens not in bold were accessible in other databases or examined by one of the authors; the * sign is used for duplicates listed in the literature but not in databases. The original spelling of the localities is kept.

\section{ACANTHACEAE}

\section{DUOSPERMA [)ayton}

quadrangulare (Klotzsch) Brummilt

32 Type of Hygrophila affinis Lindau (B, holo. t?: COI. C. K. W. iso.). Palmfontein, 28-8-1899. 1 (4) $\mathrm{m}$.

sessilifolium (Lindau) Brummitt

942 Type of Hygrophila sessilifolia Lindau (B. holo, †े; BR. COI

G. K. W. Z., iso.). Chitanda (left bank). 25-5-1900. I $150 \mathrm{~m}$

\section{LEPIDAGATHIS Willd.}

macrochila Lindau

779 (B. holo. +?; COI, K. M. W, iso.). Kusisi, Likise, 16-3-1900, $1225 \mathrm{~m}$.

\section{STROBILANTHOPSIS S.Moore}

linifolia (T.Anderson ex C.B.Clarke) Milne-Redh

830 Type of Hygrophila glutinifolia Lindau (B, holo. +?; COI. G. K. M. W, iso.). Longa-Lazingua, 18-4-1900, $1350 \mathrm{~m}$.

\section{ALLIACEAE}

\section{TULBAGHIA L.}

aequinoctialis Welw. ex Baker subsp. monantha (Engl. \& Gilg) R.B.Burb.

351a Type of Tulbaghia monamtha Engl. \& Gilg (B, holo.). Kubango, Kabindere, 30-10-1899.

\section{AMARYLLIDACEAE}

\section{AMMOCHARIS Herb}

baumii (Harms) Milne-Redh. \& Schweick.

273 Type of Crinum baumii Harms (B. holo.; BR. COI, G, M. S. Z. iso.). Kubango, Chirumbu, 14-10-1899, $1200 \mathrm{~m}$.

\section{ANACARDIACEAE}

\section{OZOROA Delile}

argyrochrysea (Engl. \& Gilg) R.Fern. \& A.Fem.

892 Type of Heeria benguellensis Engl. var. perrophila Engl. \& Gilg (B, holo. †; "BM, BR. "COI, *K, M, W, Z, iso.). Kutsi, Kapulo, 4 5-1900, 1300 m. Reference: Exell \& Mendonça (1954a: 122).

941 Type of Heeria argrrochrysea Engl. \& Gilg (B, holo. t; K. lecto., designated by Fernandes $(1966: 45)$; ${ }^{*} \mathrm{BM},{ }^{*} \mathrm{COI}, \mathrm{M} . \mathbf{W}$. iso.). Chitanda (right bank), 24-5-1900, $1150 \mathrm{~m}$.

longipes (Engl. \& Gilg) R.Fern. \& A.Fern. 512 Type of Heeria longipes Engl. \& Gilg (B. holo. +: BR. COI. K. M, W. Z. iso.). Ungombekike-Kuito, 7-12-1899. $1200 \mathrm{~m}$.

stenophylla (Engl. \& Gilg) R.Fern. \& A.Fern. 662 Type of Heeria stenophv/la Engl. \& Gilg (B. holo. +: *BM. W, Z. iso.). Longa (left bank), Lazingua, 20-1-1900. $1250 \mathrm{~m}$. Reference: Exell \& Mendonça (1954a: 117).

xylophylla (Engl. \& Gilg) R.Fem. \& A.Fem. 664 Type of Heeria rylophylla Engl. \& Gilg (B, holo. †; Z, lecto.. designated by Femandes (1966: 48); W. Z. iso.). Quiriri, Sakkemecho, 20-1-1900, $1200 \mathrm{~m}$.

\section{SE.ARSIA F.A.Barkley}

\section{kirkii (Oliv.) Moffet}

638 Type of Rhus polyneura Engl. \& Gilg (B. holo. +: *BM. *COI. K. M. W. Z, iso.). Lazingua (near mouth), 6-1-1900, $1200 \mathrm{~m}$ Reference: Exell \& Mendonça (1954a: 103).

638a Type of Rhus polyneura Engl. \& Gilg var. hylophila Engl. \& Gilg (B. holo. †). Longa. Minnesera, 1-1900.1200 m.

tenuinervis (Engl.) Moffet 744 Type of Rhus commiphoroides Engl. \& Gilg (B. holo. t: *BM. *COl. K. M. W, Z, iso.). Kuito, Onschingue Kutue, 1-3-1900, 1200 m. References: Exell \& Mendonça (1954a: 102); Kokwaro (1986: 33).

\section{ANISOPHYLLEACEAE}

\section{ANISOPHYL.LEA R.Br. ex Sabine}

quangensis Engl. ex Henriy. 808 Type of Anisophllea frusiculosa Engl. \& Gilg (B, holo. +. *BM, fragment). Quiriri, Sakkemecho, 9/11?-4-19(k), $1200 \mathrm{~m}$. Reference: Mendes (1970: 41 ).

\section{ANTHERICACEAE}

\section{ANTHERICUNL.}

liliagastrum Engl. \& (jilg 461 (B. holo. t?). Kuhango (left hank). Kuimara (left bank), 11 $1899.1100 \mathrm{~m}$ 
ANTHERICUM L. (cont.)

xylorrhizum Engl. \& Gilg

530 (B, holo. †?). Kuito (near), 12-1899, I $150 \mathrm{~m}$

\section{CHLOROPHYTUM Ker Gawl.}

colubrinum (Baker) Engl.

611 Type of Chlorophytum dolichostachys Engl. \& Gilg (B. holo.). Longa, Napalanka, 1-1-1900, 1150 m.

psammophilum Engl. \& Gilg

517 (B, holo.; COI, M, K, Z, iso.). Ungombekike-Kuito, 9.121899. $1200 \mathrm{~m}$.

\section{APIACEAE}

\section{DIPLOLOPHIUM Turcz}

zambesianum Hiem

834 Type of Physotrichia arenaria Engl. \& Gilg (B. holo. t; BM, ${ }^{*} \mathrm{COI}, \mathrm{G},{ }^{*} \mathrm{~K}, \mathbf{W}, \mathrm{Z}$, iso.). Lazingua, 19-4-1900, 1275 m. References: Cannon (1970: 350); Townsend (1989: 83).

\section{APOCYNACEAE}

\section{ASCLEPIAS L.}

baumii Schltr.

699a (B. holo. †). Longa, 3-2-1900, $1250 \mathrm{~m}$.

\section{ASPIDOGLOSSU M E.Mey.}

masaicum (N.E.Br.) Kupicha

413 Type of Schizoglossum baumii Schltr. ex N.E.Br. (K, holo.; COI, E. M. W. Z, iso.). Kubango, Kavanga, 17-11-1899. $1100 \mathrm{~m}$.

\section{CRYPTOLEPIS R.Br.}

producta N.E.Br.

457 Type of Cnptolepis haumii Schltr. (B, holo. †; BM, COI, K.

M. W. Z, iso.). Kubango (left bank). Kuimarva (left bank), 23-11$1899,1100 \mathrm{~m}$

\section{LANDOLPHIA P.Beauv.}

camptoloba (K.Schum.) Pichon

669 Type of Carpodimus leucantha K.Schum. (B, holo. +; W, lecto., designated by Persoon et al. (1992: 39); G, W, iso.). Longa, Lazingua. 23-1-1900, $1250 \mathrm{~m}$.

\section{TACAZZEA Decne}

apiculata Oliv

276 Type of Tacazzea verticillata K.Schum. (B, holo. ${ }^{*}$, COI, iso.). Kubango, Chirumbu. 10-1899. $1200 \mathrm{~m}$

rosmarinifolia (Decne.) N.E.Br.

245 Type of Tacazzea salicina Schltr. (B, holo. +: G, lecto., designated by Venter. Verhoeven \& Kotze (1990: 107); BM. COI. E. G. K. W. Z, iso.). Nambali, 6-10-1899, $1280 \mathrm{~m}$.

\section{TYLOPHORA R.Br}

caffra Meisn.

526 Type of Gymnema melananthum K.Schum. (B, holo. +; BR, COI. K. M. W. Z, iso.). Kuito (near), 12-1899, I 150 m.

\section{XYSMALOBIUM R.Br.}

undulatum (L.) W.T.Aiton

332 Type of Asclepias leucotricha Schltr. (B, holo. t; E, W, Z, iso.). Kuebe, 28-10-1899. $1150 \mathrm{~m}$.

\section{ARACEAE}

\section{ZANTEDESCHIA Spreng.}

albomaculata (Hook.) Baill. subsp. albomaculata 398 Type of Zantedeschia chloroleuca Engl. \& Gilg (B. holo. +?). Kuhango (left bank), Kucio, 11-1899, 1 120 m.

\section{ASPARAGACEAE}

\section{ASPARAGUS L.}

altiscandens Engl. \& Gilg

402 (B. holo.; COI, M. S, iso.). Kubango (left bank), Quatiri (lett hank). 12-11-1899.1100 m. baumii Engl. \& Gilg

150 (B, holo.; COl, M, iso.). Okachitanda (right margin), 25-9. 1899 .

\section{ASPHODELACEAE}

ALOE L.

metallica Engl. \& Gilg

891 (B, holo. †?). Kutsi, Kapulo, 5-1900, $1300 \mathrm{~m}$.

nuttii Baker

698 Type of Aloe brunneo-punctata Engl. \& Gilg (B, holo: B, BII BR. COI, E, K. M. iso.). Longa, Quiriri, 2-2-1900, $1250 \mathrm{~m}$.

zebrina Baker

275 Type of Aloe haumii Engl. \& Gilg (B, holo. +?; E, iso.). Kuhango, Chirumbu, 14-10-1899, I $200 \mathrm{~m}$.

\section{BULBINE Wolf}

abyssinica $A$. Rich.

487 Type of Bulbine xanthohorrys Engl. \& Gilg (B, syn.; W, isosyn.). Habungu, 28-11-1899, I 100 m.

731 Type of Bulhine xanthohotrys Engl. \& Gilg (B, syn.: BR, K M. S. W, iso.). Quiriri, Kampuluve, 22-2-19(1), $1350 \mathrm{~m}$.

\section{TRACHYANDRA Kunth}

arvensis (Schinz) Oberm.

412 Type of Anthericum pallidiflasum Engl. \& Gilg (B, holo. †?; BM, K, M, W, iso.). Kubango (left hank), Kavanga, 16-11-1899 $1100 \mathrm{~m}$

\section{ASTERACEAE}

\section{ANISOPAPPUS Hook. \& Am.}

chinensis Hook. \& Am. subsp. chinensis var. chinensis 877 Type of Anisopappus gracilis O.Hoffm. (B, holo. †; *COI, $\mathbf{K}$ M. S, W, Z iso.). Kulei-Kutsi, 29-4-1900, I 3(Y) m. Reference: Mendonça (1943: 88)

801 Type of Anisopappus subdiscoideus O.Hoffm. (B. holo. †; *COI, K. M. S. W, Z, iso.). Kampuluve, 6-4-1900), 1200 m. Reference: Mendonça (1943: 88).

\section{ARTEMISIOPSIS S.Moore}

villosa (O.Hoffm.) Schweick.

67 Type of Amphidora lasiocephala O.Hoffm. (B, holo. t; W iso.) Ediva-Humbe, Kakulovar, 6-9-1899, 1 140 m. Reference: Mendonça (1943: 61).

\section{BIDENS L}

baumii (O.Hoff̈m.) Sherf

88.3 Type of Coreopsis haumii (O.Hoffm. (B, holo, †; W, lecto. designated by Rayner (1992: 89): BM. ${ }^{*}$ COI, G, ${ }^{*}$ K, M. Z, iso.) Kuma-Kutsi, 5-19(\%), I 30) m. Reference: Mendonça (1943: 103).

flabellata O.Hoffm

847 (B. holo. +; G, lecto., designated by Rayner (1992: 82): "BM, *BR, *K. W. Z iso. Manonge, Kuebe, 22-4-1900, I $350 \mathrm{~m}$. Reference: Mendonça (1943: 102)

\section{cONYZA Less.}

limosa O.Hotfm.

358 (B, syn. +; G, isosyn.). Kubango (right bank), Kueio, 1-II1899, 1300 m. Reference: Mendonca (1943:51). $\$ 33$ (B, syn. †; BM. *COI, G, K, M, W, Z, isosyn.). Maramba Kalolo, 20-11-1899.1 100 m. Reference: Mendonça (1943:51).

welwitschii (SMore) Wild

342 Type of Nidorella limearifolia O.Hotfm. (B, holo. t). Kubango, Kabindere, 30-10-1899, I 130 m. Reference: Mendonça (1943:49).

\section{CRASSOCFPH ALUM Monch}

uvens (Hiem) S Moxore

354 Type of Senecio telmatophilus O.Hoffm. (B, holo. t; COI, BM. M. W, Z, iso.). Kubango (right bank), Kucio, $11-1899,1130 \mathrm{~m}$ Reference: Mendonca (1943: 119). 
DICOMA Cass.

anomala Sond. subsp. anomala

804 Type of Dicoma anomala Sond. var. latifolia O.Hoffm. (B, holo. †: W, iso.). Quiriri, Sakkemecho. 9-4-1900.1200 m.

933 Type of Dicoma anomala Sond. var. microphylla O.Hoffm. (B, holo. +). Malolla Katumba-Chibia, 14-6-1900, 1350 m. Reference: Mendonça (1943: 143).

antunesii $\mathrm{O}$. Hoffm.

926 (B, holo. †; *COI). Kubango-Kassinga, Mundongo, 18-51900, I $400 \mathrm{~m}$. Reference: Mendonça (1943: 140).

\section{EMIILIA Cass.}

baumii (O.Hofim.) S.Moore

707 Type of Senecio haumii O.Hofim. (B, holo. $\div$ : BM. ${ }^{*}$ COI, K. M, W, Z, iso.). Longa, Quiriri, 5-2-1900. Reference: Mendonça (1943: 112).

limosa (O.Hoffm.) C.Jeffrey

907 Type of Senecio limosus O.Hoffm. (B, holo. +; *COI, *K, W. $\mathrm{Z}$, iso.). Kubango, Kohi, 14-5-1900, $1350 \mathrm{~m}$. Reference: Mendonça (1943: 118).

\section{ERIGERON L.}

\section{baumii $O$.Hoffim}

187 (B. holo. †; BM. *COI, K. M. W. Z, iso.). Goudkopje-Kakele, 2-10-1899, 1210 m. Reference: Mendonça (1943: 46).

\section{ERLANGEA Sch.Bip}

linearifolia (O.Hoffm.) S.Moore

710 Type of Bothriocline linearifolia O.Hoffm. (B, syn. +; *COI. W, Z, isosyn.). Longa, Quiriri, 2-1900, 1275 m. Reference: Mendonça (1943: 4).

784 Type of Bothriocline linearifolia O.Hoffm. (B, syn. t; *COI,

K. M, W, Z, isosyn.). Onschingue, Kuito (near), 22-3-1900, 1200 m. Reference: Mendonça (1943:4).

\section{GRANGEA Adans.}

anthemoides $\mathrm{O}$. Hoffm

126 (B, holo. †; M. lecto., designated by Fayed (1979: 472): *COI,

K. W. iso.). Kunene and Chitanda, 21-9-1899, $1100 \mathrm{~m}$. Reference: Mendonça (1943: 43).

\section{HELICHRYSUM Mill.}

congolanum Schltr. \& O. Hoffim.

226 (B, holo. †: *COI, K, M, W, iso.). Kuelleis (Maramba), 5-101899, I 400 m. Reference: Mendonça (1943: 69).

\section{INULA L.}

limosa O.Hoffim

474 (B, syn. †). Habungu, Maramba, 28-11-1899, 1 10() m. Reference: Mendonça (1943: 77).

630 (B, syn. +; W, Z, iso.). Longa, (hijija, 5-1-1900, $1200 \mathrm{~m}$. Reference: Mendonça (1943: 77).

paludosa $\mathrm{O}$.Hoffim.

699 (B. holo. +; *COI, K. W. Z, iso.). Longa, Quiriri, 3-2-1900, I $250 \mathrm{~m}$. Reference: Mendonça (1943: 77).

\section{MELANTHER I Rohr}

triternata (Klatt) Wild

197 Type of Melamthera baumii O.Hoffm. (B, holo. + ; В М, СОI.

K. W. iso.). Goudkopje-Kakele, 3-10-1899, I 238 m. Reference: Mendonça ( 1943: 97)

\section{NICOLASIA S.More}

felicioides S.Moore

50) Type of Laggera humilis O. Hoffm. (B. holo, †; BM. *COI, W Z. iso.). Chihinde, 2-9-1899, $1270 \mathrm{~m}$. Reference: Mendonça (1943 57).

\section{NIDOREILA Cass}

\section{resedifolia [)$($. subsp. resedifolia}

47 Type of Nidorella densiflora O.Hoffm. (13, holo. +). (hihinde. 2-9-1899. I $270 \mathrm{~m}$. Reference: Mendonça (1943: 49).

\section{PASACCARDOA Kuntze}

baumii $O$.Hoffm

318 (B, holo. †: K. W. Z, iso.). Kuebe (near), 24-10-1899. 1170 m. Reference: Mendonça (1943: 139).

\section{SENECIO L}

cryphiactis $\mathrm{O}$.Hoffm.

68 (B. holo. †; COl. K. W, Z, iso.). Ediva-Humbe. Kakulovar, 6-91899. 1140 m. Reference: Mendonça (1943: 120).

\section{SPHAERANTHUS L.}

flexuosus O.Hoffm. ex De Wild.

184 Type of Sphaeranihus humilis O.Hoffm. (B. holo. †; *COI, K. M. W. iso.). Goudkopje-Kakele, 2-10-1899, 1210 m. Reference: Mendonça (1943: 61)

\section{VERNONIA Schreb}

\section{brideliifolia $\mathrm{O}$.Hoffm.}

610 (B, holo. +; W, Z, iso.). Longa. Napalanka. 1-1-1900, I $150 \mathrm{~m}$ Reference: Mendonça (1943: 30).

gerberiformis Oliv. \& Hiem subsp. macrocyanus (O.Hoffm.) C. Jeffrev 134 Type of Vernonia primulina O.Hoffm. (B, holo. +: *COI. M. W. Z, iso.). Chitanda (left bank), Kunene, 23-9-1899, 1108 m. Reference: Mendonça (1943: 29).

limosa O.Hoffm

255 (B. syn. +; G. isosyn.). Nambali. Maramba, 7-10-1899. 1250 m. Reference: Mendonça (1943: 27)

624 (B. syn. †; G. W. Z. isosyn.). Longa, Chijija. 4-1-1900, 1200 m. Reference: Mendonça (1943: 27).

mesogramme O.Hoffm

616 (B. holo. †). Kuairiri. 2-1-1900, 1150 m. Reference: Mendonça (1943: 35).

pygmaea $O$. Hoffm

235 (B. holo. †: W. iso.). Maramba, Kuelleis, 5-10-1899.1400 m. Reference: Mendonça (1943: 30).

subplumosa O.Hoffm.

703 (B, holo. †: BM, W, Z, iso.). Longa. Quiriri, 3-2-1900, 1250 m. Reference: Mendonça (1943: 10).

temnolepis O.Hoffm

929 Type of Vernonia baumii O.Hoffm. (B, syn. †; *COI. K. M, W. Z, isosyn.). Chitanda. Kassinga, 19-5-1900, $1325 \mathrm{~m}$. Reference: Mendonça (1943: 25).

929a Type of Vernonia baumii O.Hoffm. (B, syn. +: *COI, W, isosyn.). Chitanda. Kassinga, 19-5-1900, 1325 m. Reference: Mendonça (1943: 25).

\section{BORAGINACEAE}

\section{TRICHODESMA R.Br.}

baumii Gürke

844 (B, holo. ${ }^{\dagger}$; BM. COl, E, M. W. Z, iso.). Manonge. Kuebe, 22. 4-1900, $1350 \mathrm{~m}$.

\section{BRASSICACEAE}

\section{RORIPPA Scop}

cryptantha (A.Rich.) Robyns \& Boutique

129 Type of Nastursium microcapsum Engl. \& Gilg (B. holo.; BM COI, K. iso.). (Okachitanda (left bank), 22-9-1899. Reference: Exell \& Mendonça (1937a: 50).

\section{BURMANNIACEAE}

\section{BURMANYIA}

\section{madagascariensis Mar}

619 Type of Burmunmia hicolor Mart. var. micramha Engl. \& Gilg (B. holo.; C()I, S. iso.). Longa. (hijija, 4-1-1900. $1200 \mathrm{~m}$. 726 Type of Burmannia hlanda Engl. \& Gilg (B. holo.: B. K. iso.). Quiriri, Sobi. 20-2-1900.1 $300 \mathrm{~m}$. Reference: Cowlev (1988: 2). 


\section{CAMPANULACEAE}

WAHLENBERGIA Schrad. ex Roth

banksiana A.DC.

421 Type of Wahlenhergia leucantha Engl. \& Gilg (B. holo. +; K, lecto., designated by Thulin (1975: 119); BM. COI. G, K. M. ${ }^{*} \mathrm{~S}$,

W, Z, iso.). Kubango (left bank), Kavanga, 18-11-1899, $1100 \mathrm{~m}$.

denticulata (Burch.) A.DC.

837 Type of Lightfootia laricifolia Engl. \& Gilg (B. holo. †; Z, lecto., designated by Thulin (1975: 116); G. W. ${ }^{*} Z$, iso.). Lazingua, 19-4-1900, $1275 \mathrm{~m}$.

undulata (L.f.) A.DC

477 Type of Wahlenbergia cranea Engl. \& Gilg (B, holo. t; M. lecto., designated by Thulin (1975: 76); *BM, COI, G. K. ${ }^{*}$ S, W, $\mathrm{Z}$, iso.). Habungu, 28-11-1899, $1100 \mathrm{~m}$

\section{CAPPARACEAE}

\section{BOSCIA Lam}

welwitschii Gilg

999 Type of Boscia suaveolens Gilg (B, holo. †?; *BM, COI. *K. M. W, Z, iso.). Jau, 19-6-1900, I $100 \mathrm{~m}$. Reference: Exell \& Mendonça (1937b: 65).

\section{CARYOPHYLLACEAE}

POLYCARPAEA Lam.

inaequalifolia Engl. \& Gilg

816 (B, holo. †; *BM, Z, iso.). Quiriri, Sakkemecho, 4-19()0, 1200 m. Reference: Exell \& Mendonça (1937c: 112).

\section{CELASTRACEAE}

GYMNOSPORIA (Wight \& Am.) Hook.f.

senegalensis (Lam.) Loes.

565 Type of Gimnosporia haumii Loes. (B, holo. +; BM. * $\mathrm{COI}$ K. W, Z, iso.). Jonkoa, 21-12-1899, $1150 \mathrm{~m}$. Reference: Exell \& Mendonça (1954b: 8 )

\section{SALACIA L.}

bussei Loes.

480 Type of Salacia rehmanii Schinz var. haumii Loes. (B, holo. +?). Habungu, 11-1899, 1 I00) m. Reference: Exell \& Mendonça (1954b: 24)

\section{COMBRETACEAE}

\section{COMBRETUM Loefl}

\section{aureonitens Engl. \& (jilg}

53 (B. syn. +; BM. lecto., designated by Exell \& Garcia (1970: 57 ), BR. ${ }^{*}$ COI, K, M, W, Z, iso.). Chihinde-Ediva, 2-9-1899, $1250 \mathrm{~m}$ 113 (B. syn. †: BM. *COI, K. W. isosyn.). Hartebest-Lövenpan. 18-9-1899, 1 100 m. Reference: Exell \& Garcia (1970: 57).

982 (B. syn. +; *BM, *COI, K. M, W. isosyn.). Makopi, I1-6. 1900. Reference: Exell \& Garcia (1970: 57).

collinum Fresen.

54 Type of Combretum monticola Engl. \& Gilg (B, syn. †; BM lecto., designated by Exell \& Garcia (1970:58)). Chihinde-Ediva, 9-1899. $1250 \mathrm{~m}$.

56 Type of Combretum monticola Engl. \& Gilg (B, syn. +; W, Z, isosyn.). Chihinde, 2-9-1899, $1270 \mathrm{~m}$

981 Type of Combretum pachycarpum Engl. \& Gilg (B, syn. †) Makopi. 6-1900, $1200 \mathrm{~m}$

983 Type of Combretum pachycarpum Engl. \& Gilg (B, syn. +; BM. lecto., designated by Exell \& Garcia (1970): 58): BR. ${ }^{*} \mathrm{COI}$

K. M, S. W, Z, iso. ). Makopi, II-6-19(00, I 20x) m.

986 Type of Combretum laeteviride Engl. \& Gilg (B. holo. +; BM. lecto., designated by Exell \& Garcia (1970: 58); BR. *COI, K. M. S. W, Z, iso.). Meschekke-Katumba, 14-6-190(0, $1350 \mathrm{~m}$.

molle R.Br. ex G.Don

127 Type of Combrefum arbuscula Engl. \& Gilg (B, syn. †; BM COI. K. W. Z, isosyn.). Chitanda (left bank), 21-9-1899, I $100 \mathrm{~m}$. 155 Type of Combrenum arbuscula Engl. \& Gilg (B, syn. †; BM. K. W. isosyn.). Chitanda (right margin), 25-9-1899, $1150 \mathrm{~m}$.
947 Type of Combrefum arbuscula Engl. \& Gilg (B, syn. †: BM, BR. COI. K, S, W. isosyn.). Chitanda (left bank), 27-5-19(0), $1150 \mathrm{~m}$.

platypetalum Welw. ex M.A.Lawson subsp. baumii (Engl. \& Gilg) Exell

117 Type of Combrelum haumii Engl. \& Gilg (B, syn t; BM. lecto., designated by Exell \& Garcia (1970: 73); BM, COI, K. M W. Z, iso.). Hartebeest-Lövenpan, 18-9-1899, I 100 m.

119 Type of Combrenum haumii Engl. \& Gilg (B, syn. †). Hartebeest-Lövenpan, 9-1899, $1100 \mathrm{~m}$.

232 Type of Combretum arenarium Engl. \& Gilg (B, holo. †; BM. lecto., designated by Exell \& Garcia (1970: 72); BM. K. W. Z, iso.). Kuelleis (Kassinga-Kubango), 5-10-1899, $1400 \mathrm{~m}$.

232a Type of Combretum argurochryseum Engl. \& Gilg (B, holo. †; BM, fragment and drawing of holo. [cited by Exell \& Garcia (1970) 72)]. W, iso.). Kuelleis (Kassinga-Kubango), 5-10-1899, $1400 \mathrm{~m}$. 232b Type of Combretum gnidioides Engl. \& Gilg (B, holo. †; BM. lecto., designated by Exell \& Garcia (1970: 72); W. Z, iso.). Kuelleis (Kassinga-Kubango), 5-10-1899, $1400 \mathrm{~m}$.

psidioides Welw. subsp. dinteri (Schinz) Exel

722 Type of Combretum quirirense Engl. \& Gilg (B, holo. + ; BM lecto., designated by Exell \& Garcia (1970: 66); BR. *COI. ${ }^{*} \mathrm{~K}, \mathrm{~S}$ W, Z, iso.). Quiriri, Sobi, 19-2-1900, $1300 \mathrm{~m}$.

\section{TERMINAL.LA L.}

brachystemma Welw. ex Hiern

215 Type of Terminalia haumii Engl. \& Gilg (B, holo, +: BM lecto., designated by Exell \& Garcia (1970: 89); ${ }^{*}$ COI, K. M, W. $Z$, iso.). Kassinga-Kolove, 4-10-1899, $1300 \mathrm{~m}$.

\section{COMMELINACEAE}

ANEILEMA R.Br.

plagiocapsa K.Schum 716 (B. holo,; BM, C(), G, K. W. iso.). Quiriri, Sobi, 18-2-1900, $300 \mathrm{~m}$

\section{COMMELINA L}

\section{milne-redheadii Faden}

256 Type of Commelina welwitschii C.B.Clarke var, glabra K.Schum. (B, holo. t: B.M. K. iso.). Nambali, 7-10-1899, $1250 \mathrm{~m}$.

subulata Roth

963 Type of Commelina eleganrula K.Schum. (B, holo, B W. M, iso.). Kitewe-Humbe, 3-6-1900, I 100 m.

\section{welwitschii C.B.Clarke}

814 Type of (ommelina filifolia K.Schum. (B. holo,; K. iso.). Quiriri, Sakkemecho, 15/16?-4-19(K), I 200) m

\section{FLOSCOPA Lour.}

flavida C.B.Clarke

915 Type of Floscopa pusilla K.Schum. (B, holo, †?; BR. K. M, S, iso.). Kubango, Kohi, 15-5-19(00.

\section{CONVOLVULACEAE}

\section{IPOMOEA I}

crassipes Hook.

151 Type of Ipomoea crassipes Hook. var. hirla Hallier f. (B. syn. †). Chitanda (right margin), 25-9-1899. I $150 \mathrm{~m}$

454 Type of Ipomoea crassipes Hook. var. hirla Hallier f. (B. syn. †; COI, M. W. iso.). Kuhango (left bank), Kuimarna, 23-11-1899, $1100 \mathrm{~m}$

\section{CRASSULACEAE}

\section{KILANCHOE Adans}

brachyloba Welw, ex Britten

938 Type of Kalanchoe batumii Engl. \& Gilg (B, holo, †). Goudkopje, 22-5-19(0), I 300 m.

\section{CUCURBITACEAE}

\section{TROCHOMERII Hook.f.}

\section{baumiana Gilg}

445 (B. holo. +), Kubango (left bank), Kalolo, $11-1899.1100 \mathrm{~m}$. 


\section{CYPERACEAE}

\section{CYPERUS L.}

chrysocephalus (K.Schum.) Kük.

311 Type of Mariscus chrysocephalus K.Schum. (B, syn. †?; K. isosyn.). Longa, Maschonge, 23-11-1899, $1170 \mathrm{~m}$.

311 a Type of Mariscus chrysocephalus K.Schum. (B. syn. +?; COI. isosyn.). Longa, Maschonge, 31-12-1899, $1150 \mathrm{~m}$.

\section{RHYNCHOSPORA Vahl}

holoschoenoides (Rich.) Herter

355 Type of Rhynchospora dolichostyla K.Schum. (B, holo. +?; K iso.). Kubango (right bank), Kueio, 1-9-1899, I I30 m.

\section{DICHAPETALACEAE}

\section{DICHAPETALUM Thouars}

cymosum (Hook.) Engl.

64 Type of Dichaperalum venenatum Engl. \& Gilg (B, holo. t; $M$, lecto., designated by Breteler (1978: 32); BM. BR. COI. E. K. M. $\mathbf{S}, \mathbf{W}, \mathbf{Z}$, iso.). Ediva-Humbe, 6-9-1899, $1200 \mathrm{~m}$.

\section{DIPSACACEAE}

\section{CEPHALARIA Schrad.}

retrosetosa Engl. \& Gilg

894 (B, holo. †: BM, COI, E, K, Z, iso.). Kutsi, Kapulo, 5-5-1900, $1300 \mathrm{~m}$.

\section{DIPTEROCARPACEAE}

\section{MONOTES A.DC.}

\section{dasyanthus (iilg}

888 (B, holo. †; *BM, *COl, K. M. W, Z, iso.). Kutsi, 2-5-1900), $1300 \mathrm{~m}$. Reference: Bancroft (1937: 139).

\section{EBENACEAE}

\section{DIOSPY ROS L.}

batocana Hicrn

780) Type of Diospyros xamthocarpa Gürke (B, holo. + ; W, iso.). Ḱusisi, Likise, 16-3-1900, $1200 \mathrm{~m}$.

\section{kirkii Hiern}

945 Type of Diospıros haumii Gürke (B, holo. +: BR. K. M. W, Z iso.). Chitanda (left bank), 26-5-1900, $1150 \mathrm{~m}$.

virgata (Gürke) Brenan

792 Type of Maha vingata Gürke (B. holo. †: G, iso.). Onschingue. Kuito (near), 1-4-1900, I $200 \mathrm{~m}$.

\section{EUCLEA Murtay}

crispa (Thunb.) Gürke

191 Type of Euclea haumii Gïrke (B. holo. †: COI. K. M, W, Z. iso.). Goudkopje-Kakele, 10-1899, $1210 \mathrm{~m}$

\section{divinorum Hiern}

33 Type of Euclea huillensis Gürke (B, holo. ${ }^{+}$: BR. COI, K. M. S. W. Z. iso.). Palmfontein. Ediva, 28-8-1899. I ( $(x) \mathrm{m}$.

\section{ELATINACEAE}

\section{BERGIA L.}

pentheriana Kéissl.

98 Type of Bergia pallide-rosea Gilg (B, holo, t; *BM, BR. COI, M. S. W, iso.). Kunene (left bank). Humbe, 14-4-1899, 1 $140 \mathrm{~m}$. Reference: Exell \& Mendonça (1937d: 118).

\section{spathulata Schinz}

110 Type of Bengia ernthrolenca (iilg (B, holo. +: *BM, BR. C(OI. K. M. S. W, Z, iso, ). Kunene (left bank), Soba Gongo, 17-9-1899. I 115 m. Reference: Exell \& Mendonça (1937d: 119).

\section{EUPHORBIACEAE}

\section{JATROPHA L.}

baumii Pax

122a (B. holo. +")). Löwenpan-Kunene, 19-9-1899. $1100 \mathrm{~m}$.

\section{SCLEROCROTON Hochst.}

oblongifolius (Müll.Arg.) Kruijı \& Roebers

257 Type of Sapium suffruticosum Pax (B, holo. +?; BR. COI, E.

K. M. W, iso.). Nambali. Maramba. 10-1899, $1250 \mathrm{~m}$.

\section{FABACEAE}

\section{ADENODOLICHOS Harms}

baumii Harms

848 (B. holo. †; COl, lecto., designated by Torre (1966: 301): BM. COI, "K. M. Z, iso.). Manonge, 22-4-1900, $1350 \mathrm{~m}$.

rhomboideus (O.Hoffm.) Harms var. rhomboideus

872 Type of Adenodolichos euryphyllus Harms (B. holo. +; *COI, BM, K. Z. iso.). Kulei-Kutsi, 29-4-1900, 1300 m. Reference: Torre (1966: 300).

\section{AESCHYNOMENE L.}

\section{baumii Harms}

810 (B. holo. +; COI, lecto., designated by Torre (1966: 198); BR. COI, ${ }^{*}$ K, M. S. W. Z, iso.). Quiriri. Sakkemecho, 10-4-1900, 1200 $\mathrm{m}$

bracteosa Welw. ex Baker 252 Type of Aeschinomene nambalensis Harms (B. holo. t; BM. *COI, ${ }^{*} \mathrm{~K}$. W, Z, iso.). Nambali, 7-10-1899, 1250 m. Reference: Torre (1966: 199).

\section{BAIKIAEA Benth.}

plurijuga Harms 428 (B, holo. t: *BM. COI. K. M. W, Z. iso.). Kubango (left bank), Kalolo, 20-11-1899, 1100 m. Reference: Torre \& Hillcoat (1956: 220).

\section{BAPHIA Afzel. ex Lodd.}

massaiensis Taub. subsp. obovata (Schinz) Brummitt var. cornifolia (Harms) Soladoye

121 Type of Baphia cornifolia Harms (B, holo. t; K. lecto., designated by Hillcoat (1966: 376); BM, *COI, ${ }^{*}$ K, M. W, Z, iso.). Lövenpan-Kunene, 18-9-1899. $1100 \mathrm{~m}$.

\section{COPAIFERA L.}

\section{baumiana Harms}

523 (B, holo. †: *BM. COI. K, M, S. W, Z, iso.). UngombekikeKuito, 10-12-1899, I $200 \mathrm{~m}$. References: Torre \& Hillcoat (1956: 243).

\section{CROTALARIA L.}

angulicaulis Harms

740 (B. holo. †: BM. iso.). Kampuluve, 2-1900, $1200 \mathrm{~m}$.

baumii Harms

836 (B. holo. t: BM. COI. K. W. M. S. Z. iso.). Lazingua, 19-41900, 1275 m. Reference: Torre (1962:35).

densicephala Welw. ex Baker 670 Type of Crofalaria lachnoclada Harms (B. holo. +: BM. COI K. M, S. W, Z, iso.). Longa. Lazingua. 23-1-1900. $1250 \mathrm{~m}$. Reference: Torre (1962: 63).

leptoclada Harms var. leptoclada

829 (B. holo. †: BM. COI. K. LISC. M, W. Z, iso.). Longa, 18-41900). 1275 m. Reference: Torre (1962: 66)

psammophila Harms

741 (B, holo. †: BM. COI. K. M. S. W, Z, iso.). Kampuluve, Kuito (near), 27-2-1900.12(10) m. Reference: Torre (1962: 29).

sericifolia Harms

483 (B, holo. †; BM. COI, K. M. W, Z, iso.). Habungu, 28-11 $1899.1100 \mathrm{~m}$. Reference: Torre (1962:67).

subsessilis Harms

787 (B, holo. †. BM. COI, K. M, W. Z. iso.). Onschingue. Kuito (near). 29-3-1900.1 2(10 m. Reference: Torre (1962:67).

EVTADA Adans.

arenaria Schinz subsp. arenaria

471 Type of Eniada nuna Harms (B, holo. +: W, Z, iso.). Habungu. $28-11-1899,1100 \mathrm{~m}$ 


\section{ERYTHRINA L.}

baumii Harms

223 (B, holo. †; BM. lecto., designated by Torre (1966: 248); E NY. W, iso.). Kuelleis (Maramba), 5-10-1899, $1400 \mathrm{~m}$.

\section{INDIGOFERA L.}

baumiana Harms

497 (B, syn. +; BM, BR, COI, E, K, M, W, Z, isosyn.). Habungu, 1-12-1899, I $150 \mathrm{~m}$. Note: Torre (1962:118) referred to the B specimen as holotype (because it was the first of the two syntypes cited in the protologue) which would make it a lectotype. However, he did not see the specimen (having referred to it as n.v.). Therefore, we think this cannot be considered a lectotypification. 819 (B, syn. †). Quiriri, Sakkemecho, 4-1900, $1200 \mathrm{~m}$.

griseoides Harms

745 (B, holo. †; E, W, iso.). Kuito, Onschingue-Kutue, 1-3-1900, $1200 \mathrm{~m}$.

nambalensis Harms

254 (B, holo. †). Nambali, 10-1899, $1250 \mathrm{~m}$.

\section{JULBERNARDIA Pellegr.}

paniculata (Benth.) Troupin

732 Type of Berlinia baumii Harms (B, holo. †; BM, COI. K. M iso.). Onschingue, Kuito, 22-2-1900, $1200 \mathrm{~m}$. Reference: Torre \& Hillcoat (1956: 212).

\section{PHYSOSTIGMA Balf.}

\section{mesoponticum Taub.}

133 Type of Physostigma mesoponticum Taub. var. haumii Harms (B, holo. +; COl, lecto., designated by Torre (1966: 260): *BM, E, K. iso.). Chitanda (left bank), 23-9-1899, $1108 \mathrm{~m}$.

\section{RHYNCHOSIA Lour.}

baumii Harms

764 (B, holo. +; Z, lecto., designated by Torre (1965: 223); S. W. Z, iso.). Kuito, Kutue-Sobi, 4-3-1900, $1200 \mathrm{~m}$

Iuteola (Hiem) K.Schum

1005a Type of Rhynchosia moninensis Harms (B. holo. + $\mathrm{BM}$, ${ }^{*} \mathrm{COI},{ }^{*} \mathrm{~K}, \mathbf{W}, \mathrm{Z}$, iso.). Monino, Nevis, 23-6-1900, $350 \mathrm{~m}$. Reference: Torre (1966: 316)

\section{SESBANIA Scop.}

coerulescens Harms

782 (B. holo. †; K. M, W. Z, iso.). Miané, Kavamba, 14-3-1900, $1175 \mathrm{~m}$. Reference: Sousa (1962: 184).

\section{microphylla Harms}

569 (B, holo. t: K. Z, iso.). Longa (mouth), 22-12-1899, I $130 \mathrm{~m}$ Reference: Sousa (1962: 186)

\section{TEPHROSIA Pers.}

cephalantha Welw. ex Baker

563 Type of Tephrosia hypangirea Harms (B, holo. t; "BM, COI, ${ }^{*} \mathrm{~K}$, M. W. Z, iso.). Jonkoa, 20-12-1899, 1150 m. Reference: Torte (1962: 164).

\section{coronilloides Welw. ex Baker}

612 Type of Tephrosia longana Harms (B, holo. †; BM. COI. K W. Z, iso.). Longa, Napalanka, 1-1-1900, $1150 \mathrm{~m}$. Reference: Torre (1962: 155).

\section{FLACOURTIACEAE}

\section{XYLOTHECA Hochst.}

\section{longipes (Gilg) Gilg}

388 Type of Oncoba longipes Gilg ( B, holo. +: G, iso.). Kubango (right bank), Kueio, 5-11-1899, $1100 \mathrm{~m}$. Reference: Sleumer (1937: 81).

\section{GENTIANACEAE}

\section{CHIRONIA L.}

baumiana Gilg

739 (B, holo. †; COI. E, K. M. W, Z, iso.). Kampuluve, 26-2-19(1), $1210 \mathrm{~m}$.
NEUROTHECA Salisb. ex Benth.

congolana De Wild. \& T.Durand

604 Type of Neurotheca baumii Gilg (B, holo. †; COI, E. K. M. S, W, Z, iso.). Longa, Napalanka, 1-1900, I $150 \mathrm{~m}$.

\section{PYCNOSPHAERA Gilg}

buchananii (Baker) N.E.Br.

752 Type of Pycnosphaera irimera Gilg (B, holo. +; COI, E, K, M

S, W, Z, iso.). Kuito, Kutue, 2-3-1900, $1200 \mathrm{~m}$.

\section{SEBAEA Sol. ex R.Br.}

baumiana (Gilg) Boutique

692 Type of Belmontia haumiana Gilg (B, holo. +; COI, E. W, Z, iso.). Longa, Quiriri, 2-2-1900, $1250 \mathrm{~m}$.

teuszii (Schinz) P.Taylor

316 Type of Belmontia chionantha Gilg (B, syn. +; ${ }^{*} \mathrm{~K}$, isosyn.). Kuebe (near), 10-1899, 1170 m. Reference: Nemomissa (2002: 18).

702 Type of Belmontia chionantha Gilg (B, syn. +; E, *K, W, isosyn.). Longa, Quiriri, 3-2-1900, $1250 \mathrm{~m}$. Reference: Nemomissa (2002: 18)

838 Type of Belmontia chionantha Gilg (B, syn. †; COl, E, K. M. W. Z, isosyn.). Lazingua, 19-4-1900, I 300 m. Reference: Nemomissa (2002: 18)

\section{HYACINTHACEAE}

\section{DIPC ADI Medik}

longifolium (Lindl.) Baker 499 Type of Dipcadi anthericoides Engl. \& Gilg (B. holo.; COI. K. M. iso.). Bondo, 2-12-1899, $1150 \mathrm{~m}$.

Vaginatum Baker

349 Type of Dipcadi haumii Engl. \& Gilg (B. holo.). Kubango, Kabindere, 30-10-1899, $1150 \mathrm{~m}$.

viride (L.) Moench

459 Type of Dipcadi lividescens Engl, \& Gilg (B, holo.). Kubango (left bank), Kuimarva, 23-11-1899, $1100 \mathrm{~m}$.

\section{LEDEBOURIA Roth}

revoluta (L.f.) Jessop

203a Type of Scilla lanceifolia (Jacq.) Baker var. longiracemosa Engl. \& Gilg (B, syn. †?). Kakele, Kassinga, 10-1899, $1250 \mathrm{~m}$. 447 Type of Scilla lanceifolia (Jacq.) Baker var. longiracemosa Engl. \& Gilg (B. syn. †?). Kubango (left bank), Kalolo, 11-1899. $1100 \mathrm{~m}$

SCHIZOC ARPHUS Van der Merwe

nervosus (Burch.) Van der Merwe

410 Type of Scilla eriospermoides Engl. \& Gilg (B, holo.; COI. K.

M. iso.). Kubango (left bank), Kavanga, 16-11-1899, I I(1)( $\mathrm{m}$.

SCILLA L.

baumiana Engl. \& Gilg

122 (B, holo.; COI, K, Z, iso.). Lövenpan-Kunene, 19-9-1899, $1100 \mathrm{~m}$.

\section{HYDROCHARITACEAE}

\section{OTTELIA Pers.}

kunenensis (Gürke) Dandy

962 Type of Boortia kumenensis Gürke (B. holo. t?; COI. K. M. W. iso.). Kunene, Kitewe-Humbe, 3-6-19(x), I 100 $\mathrm{m}$.

muricata (C.H.Wright) Dandy

772 Type of Boofria aschersoniana Gürke (B, holo. †?; COI, M. W, iso.). Kuito, Kutue-Sobi, I()-3-19(x), I $2(1) \mathrm{m}$

ulvifolia (Planch.) Walp

120 Type of Orselia benguellensis Gürke (B, holo. +?; W, iso.). Lövenpan, 18-9-1899, $11(x) \mathrm{m}$

858 Type of Ottelia baumii Gürke (B, holo. t?: (CI, K. W, iso.) Manonge, 23-4-19(0). $1350 \mathrm{~m}$. 


\section{HYDROSTACHYACEAE}

\section{HYDROSTACHYS Thouars}

triaxialis Engl. \& Gilg

263 (B, holo. †; B M, COI, K, M, W, Z, iso.). Kubango, Massaca, $8-10-1899,1200 \mathrm{~m}$

\section{HYPERICACEAE}

\section{HYPERICLM L.}

\section{lalandii Choisy}

909 Type of Hypericum haumii Engl. \& Gilg (B, holo. †). Kubango, Kohi, 5-1900, 1350 m. References: Exell \& Mendonça (1937e: 120); Milne-Redhead (1953: 9)

\section{IRIDACEAE}

\section{GLADIOL.US L.}

benguellensis Baker 632 Type of Gladiolus longanus Harms (B, holo.; BM. E, Z, iso.) Longa, Chijija, 5-1-1900, $1200 \mathrm{~m}$.

magnificus (Harms) Goldblatt 651 Type of Antholyza magnifica Harms (B. holo.; COI. M. W, iso.). Longa, Minnesera, 11-1-1900, $1250 \mathrm{~m}$.

\section{pallidus Baker}

881 Type of Gladiolus baumii Harms (B. holo.). Kulei-Kutsi, 304-1900, $1300 \mathrm{~m}$.

898 Type of Gladiolus kubangensis Harms (B. holo.: COI. E. M. S. Z, iso.). Kubango (near), 8-5-1900), $1450 \mathrm{~m}$

\section{IXONANTHACEAE}

\section{PHYLLOCOSMUS Klotzsch}

lemaireanus (De Wild. \& T.Durand) T.Durand \& H.Durand 712 Type of Phyllocosmus candidus Engl. \& (jilg (B. holo. †: BM. COI, K, W, Z, iso.). Quiriri. Sakkemecho, 13-2-1900, $1300 \mathrm{~m}$ References: Exell \& Mendonça (1951a: 247); Verdcourt (1984: 4).

\section{LAMIACEAE}

\section{AEOLLANTHUS Mart. ex K.Spreng.}

engleri Briq.

737 Type of Aeolanthus paludosus Gürke (B. holo. +: COI. E. K W, Z, iso.). Kampuluve, 26-2-1900, I $210 \mathrm{~m}$

866 Type of Aeolanthus uliginosus Gürke (B. holo. t; COI. F. K. M, S. W, iso.). Chimpungu, 25-4-1900, $1400 \mathrm{~m}$.

\section{BENGUELLIA G.Taylor}

lanceolata (Gürke) G. Taylor

910 Type of Orthosiphon lanceolatus Gürke (B. holo. †; COI, M. W, iso.). Kubango, Kohi. 15-5-1900. $1350 \mathrm{~m}$.

\section{CLERODENDRUM L.}

baumii Gürko

661 (B, holo. †; E, W, Z, iso.). Longa (left hank), Lazingua, 20)-1$1900,1250 \mathrm{~m}$

\section{PLATOSTOMA P.Beauv.}

\section{strictum (Hiern) A.J. Paton} 644 Type of Geniosporum haumii (jürke (B. holo. +: BM. COl. K. M. S. W. iso. ). Lazingua, near Longa, 7-1-19(x), $1130 \mathrm{~m}$

\section{PLECTRANTHUS L'Her.}

baumii (jürke

283 (B. holo. †: COI. W, Z, iso.). Kubango, Massaca, 19-10-1849. $1150 \mathrm{~m}$.

\section{betonicifolius Baker}

544 Type of Colerus haumii Gürke (B. holo, t: BM, СОІ, K. M, S. W. Z, iso.). Kuito, 14-12-1899, $1150 \mathrm{~m}$.

\section{guerkei Bric.}

789 lype of Hyptis baumii Gürke (B, holo, t: COl. E. M. W, Z iso.). Onschingue. Kuito (near), 1-4-1900, $1200 \mathrm{~m}$.
TINNEA Kotschy \& Peyr.

benguellensis Gürke 697 (B, holo. †; BM, COI, E, K. M. W, Z, iso.). Longa, Quiriri, 2-2-1900, $1250 \mathrm{~m}$.

coerulea Gürke var. coerulea

857 (B. holo. †; BM. G, K. M. W, Z, iso.). Manonge, Kuebe, 23 4-1900, $1350 \mathrm{~m}$.

vestita Baker

695 Type of Tinnea fusco-luteola Gürke (B. holo. † BM. COI, E, G. K. M, W, Z, iso.). Longa, Quiriri , 2-2-1900, $1250 \mathrm{~m}$.

\section{LENTIBULARIACEAE}

\section{UTRICULARIA L.}

spiralis $\mathrm{Sm}$

307 Type of Liricularia haumii Kamienski (B, holo. †; *BM, *BR COI. G. ${ }^{*} \mathrm{HBG}, \mathbf{K},{ }^{*} \mathrm{M},{ }^{*} \mathrm{~S}, \mathbf{W}, \mathrm{Z}$, iso.). Kuebe (near), 23-10-1899, $1170 \mathrm{~m}$. Reference: Taylor (1973: 8).

subulata $L$.

691 Type of Urricularia angolensis Kamienski (B. holo. + : $\mathbf{K}$. iso.). Longa. Quiriri , 2-2-1900, $1250 \mathrm{~m}$.

\section{LOBELIACEAE}

LOBE.LIA L

angolensis Engl. \& Diels ex Diels

43 Type of Lobelia minuridentara Engl. \& Gilg (B. holo.; *BM. COI, G, *K, M, *S, W, Z, iso.). Chihinde, 2-9-1899, 1270 m. Note: although the holotype has been reported as destroyed, e.g. Thulin (1984: 45), it has recently been found.

\section{MONOPSIS Salisb}

zeyheri (Sond.) Thulin

144 Type of Lobelia fonticola Engl. \& Gilg (B. holo. +: G. lecto. designated by Thulin (1979: 135): *BM. COI. K. M, S. W, iso.). Chitanda (left bank). 24-9-1899. Reference: Thulin (1984; 49).

\section{LORANTHACEAE}

\section{AGELANTHUS Tiegh}

terminaliae (Engl. \& Gilg) Polhill \& Wiens

519 Type of Loranihus terminaliae Engl. \& Gilg (B. holo; $\mathbf{K}$ Z, iso.). Ungombekike-Kuito, 9-12-1899, $1200 \mathrm{~m}$. Reference: Gebauer (1993).

\section{PHRAGMANTHERA Tiegh.}

baumii (Engl. \& Gilg) Polhill \& Wiens

217 Type of Loranthus haumii Engl. \& Gilg (B. holo.; COI, K. Z. iso.). Kassinga-Kolove, 4-10-1899, $1300 \mathrm{~m}$. Reference; Gebauer (1993)

\section{LYTHRACEAE}

\section{NESAEA Kunth}

baumii Koehne

204 (B. holo. t: COI. lecto.. designated by Femandes \& Femandes (1970: 185): K. M. W. Z. iso.). Kakele. 3-10-1899.1250 m.

\section{MALPIGHIACEAE}

SPHEDAMYOC ARPUS Planch. ex Benth. \& Hook.f.

angolensis (A.Juss.) Planch. ex Oliv.

588 Type of Sphedamnocarpus pulcherrimus Engl. \& Gilg (B. holo. t; "BM. BR. "COI. K, M. W. Z, iso.). Longa. Napalanka, 28-12-1899, $1150 \mathrm{~m}$. Reference: Exell \& Mendonça (1951b: 252).

\section{MALVACEAE}

\section{PAYONIA Cai}

senegalensis (Cav.) Leistner

760 Type of Hibiscus haumil Gürke (B. holo, +: BM. COI. "K

M. W. Z, iso.). Kuito, Kutue-Sobi, 4-3-19(0), $1200 \mathrm{~m}$. Reference: Exell \& Mendonça (1937f: 157). 


\section{MELIACEAE}

\section{ENTANDROPHRAGMA C.DC.}

ekebergioides (Harms) Sprague

88 Type of Wulfhorstia ekebergioides Harms (B, holo. †?; *BM, COI. E, K. M, W、 iso.). Humbe, 10-9-1899, 1200 m. Reference: Exell \& Mendonça (1951 c: 309).

\section{MENISPERMACEAE}

\section{TINOSPORA Miers}

caffra (Miers) Troupin

540 Type of Desmonema pallide-aurantiacum Engl. \& Gilg (B, holo.; "BM, BR, COI, E, G, K, M. S, Z, iso.). Kuito, Onjimba, 1312-1899. I 150 m. Reference: Exell \& Mendonça (1937g: 39).

\section{NYMPHAEACEAE}

\section{NYMPHAEA L.}

sulphurea Gilg

325 (B. syn.; Z, isosyn.). Kuebe (near), 26-10-1899, 1 150 m. Reference: Exell \& Mendonça (1937h: 48).

657 (B. syn.; *BM, *COI, K, isosyn.). Longa (left bank), Minnesera, 17-1-1900, 1250 m. Reference: Exell \& Mendonça (1937h: 48).

\section{OCHNACEAE}

\section{OCHNA L}

arenaria De Wild. \& T.Durand

813 Type of Ochna roseiflora Engl. \& Gilg (B, lecto., designated by Exell \& Mendonça (195Id: 285) $;$ K. W, isolecto.). Chimpungu-Kulei, 11-4-1900, 1350 m. Note: Exell \& Mendonça (1951d: 285) cited the two syntype collections 813 and $813 \mathrm{a}$, but referred to the B duplicate of no. 813 as type, which is thus the lectotype. However, this specimen was destroyed. Verdcourt (2005) cited Baum 813 (B) as holotype.

813a Type of Ochna roseiflora Engl. \& Gilg (B. syn. †; BM. E, isosyn.). Quiriri, Sakkemecho, 11-4-1900, 1200 m. Reference: Exell \& Mendonça (1951d: 285).

cinnabarina Engl. \& Gilg

550 (B, holo. \$; E, W. Z, iso.). Kuito, Longa, 16-12-1899, 1150 m Reference: Exell \& Mendonça (1951d: 292).

pulchra Hook.

114? Type of Ochna antunesii Engl. \& Gilg (B. holo. +; COI. K. P. W. Z, iso.). Hartebeest-Lövenpan, 18-9-1899, I I00 m. Note: although the description in Warburg (1903) is based on this collection, the name had been previously considered a synonym by Tieghem (see Exell \& Mendonça 195Id: 291) and the type may be a specimen collected by Antunes.

145 Type of Ochna brunnescens Engl. \& Gilg (B, holo t; BM, COI, E, K, P, Z, iso.). Chitanda (left hank), 24-9-1899, $1150 \mathrm{~m}$. Note: the name had been previously considered a synonym by Tieghem (see Exell \& Mendonça 1951d: 291).

\section{pygmaea Hiern}

172 Type of Ochna hoepfneri Engl. \& Gilg (B. lecto., designated by Exell \& Mendonça (1951d: 287)†; BM. BR. "COI. K. M, P. W. iso.). Chitanda (right margin), 27-9-1899, $1150 \mathrm{~m}$. Note: Exell \& Mendonça (1951d: 287) cite the two syntypes 172 and 220 but designate the $\mathrm{B}$ duplicate of the former as type (i.e. lectotype). This was apparently destroyed.

220 Type of Ochna hoepfneri Engl. \& Gilg (B, syn. †: BM. COI. K. M. P. W. Z, isosyn.). Kuelleis (Maramba), 5-10-1899, I 4(1) $\mathrm{m}$. Reference: Exell \& Mendonça (1951d: 287).

\section{SAUVAGESIA L}

africana (Baill.) Bamps

620 Type of Vausagesia hellidifolia Engl. \& Gilg (B, holo. t; BM. CO1, E, G, K, M. W. Z, iso.). Longa, (hijija, 4-1-190(), $1200 \mathrm{~m}$ Reference: Exell \& Mendonça (1951d: 284).

\section{ONAGRACEAE}

\section{LUDWIGIA L.}

\section{senegalensis (DC.) J.Troch.}

97 Type of Luchigia pulvinaris Gilg (B. holo. +: COI. lecto., designated by Fernandes \& Femandes (1970): 196); G, K, M, W, Z. iso.). Kunene (left bank). Humbe, 12-9-1849, 1 (1)66 m.

\section{ORCHIDACEAE}

\section{BRACHYCORYTHIS Lind!.}

angolensis (Schltr.) Schltr.

326 Type of Platanthera angolensis Schltr. (B, holo. +: BM. *COI, $\mathbf{E}, \mathbf{K}, \mathbf{M}, \mathbf{S}, \mathbf{W}$, iso.).

Kuebe, 26-10-1899, I 150 m. Reference: Summerhayes (1968: 21). 614 Type of Brachicorvthis oligophylla Kraenzl. (B, holo. †; BM, W, iso.). Longa, Napalanka, $1-1-1900,1150 \mathrm{~m}$.

rhodostachys (Schltr.) Summerh.

381 Type of Platanthera rhodostachis Schltr. (B, syn. t: "BM, E, W. isosyn.). Kubango, Kueio, 4-11-1899, $1120 \mathrm{~m}$. Note: Summerhayes (1968: 18) cited the collection 381 at B as holotype. In fact, there were two syntypes, 381 and 629 . The 381 duplicate cited as holotype was also reported as destroyed, therefore this is not considered a lectotypification.

629 Type of Platanthera rhodostachys Schltr. (B, syn. †: W, isosyn.). Longa, Chijija, 5-1-1900, $1200 \mathrm{~m}$.

\section{EULOPHIA R.Br. ex Lindl.}

adenoglossa (Lindl.) Rchb.f.

505 Type of Eulophia haumiana Kraenzl. (B. holo. + ?; K. W, iso.). Ungombekike, 5-12-1899, $1200 \mathrm{~m}$. Note: the holotype is cited by Cribb (1989: 455) as seen; however, it is not available in Aluka nor in the $B$ website.

arenicola Schltr.

384 (B, holo. †: COI, E. K. M, W. iso.). Kubango, Kueio, 11-1899. $1120 \mathrm{~m}$.

\section{calantha Schltr.}

382 Type of Eulophia calantha Schltr. var. kuhangensis Schltr. (B. holo. †). Kubango, Kueio, 4-11-1899, 1 120 m. Reference: Cribh (1989: 435).

631 (B, holo, †: BM, COI, E, ${ }^{\star} G,{ }^{*} \mathrm{~K}, \mathbf{M}, \mathbf{S}, \mathbf{W}$, iso.). Longa, Chijija. 5-1-1900, $1200 \mathrm{~m}$. Reterence: Cribb (1989: 434).

coeloglossa Schltr.

367 (B, holo, +: BM. COl, E, *G, K, M. S, W, iso.). Kubango, Kueio, 11-1899, I 120 m. Reference: Cribb (1989: 435).

corymbosa Schltr.

377 (B, holo. +: E, K, M, S. W. iso.). Kubango, Kueio, 4-1 1-1899, $1120 \mathrm{~m}$.

gonychila Schltr.

261 (B. holo. †). Nambali, Kubango (near), 7-10-1899, $1200 \mathrm{~m}$. Reference: Cribb (1989: 471).

malangana (Rchb.f.) Summerh

496 Type of Eulophia warhuryii Schltr. (B. holo. t; (C), E. K. I. S, W, iso.). Habungu, 30-11-1899, $1100 \mathrm{~m}$. Reference: Cribh (1989: 434).

rolfeana Kraenzl.

369 (B. holo. †). Kubango (right bank), Kucio (mouth), 3-11-1899.

tricristata Schltr

357 ( B, holo. t; COI, K. M, S, W. iso.). Kubango, Kabindere, 30. $11-1899.1130 \mathrm{~m}$

trilamellata De Wild

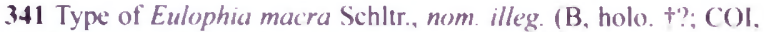
K. M. S. W. ${ }^{*} Z$, iso, ). Kubango, Kabindere. $10-1899,1130 \mathrm{~m}$. Note: the holotype is cited by Cribb (1989: 455) as seen; however, it is not available in Aluka nor in the $B$ website.

\section{HABENARI I Willd}

holothrix Schltr. 578 (B, holo. *; BM, CO), K, M, ${ }^{\star} S, \mathbf{W}, 2$, iso.). Longa, Napalanka, 12-1899, 1 150 m. Reference: Summerhayes (1968: 122).

holubii Rolfe

598 Type of Habenaria rhopaloceras Schltr. (B, holo. t: BM. "COI, K. M. W, Z, iso.). Maschonge, Napalanka, 28-12-1899, $1150 \mathrm{~m}$. Reference: Summerhayes (1968: 110).

huillensis Rchb.f 425 Type of Hahenaria kuhangensis Schltr. (B, holo, t: BM. M S. W. Z, iso.). Kuhango, Kavanga Kalolo, 19-11-1899, 1110) $\mathrm{m}$.

macroplectron Schltr 603 (B, holo, t; BM, K. M, S, W, Z, iso.). Longa, Napalanka, I- 1I $(x) .1150 \mathrm{~m}$. 
HABENARIA Willd. (cont.)

verdickii (De Wild.) Schltr.

561 Type of Habenaria monophylla Schltr., nom. illeg. (B. holo. t; BM, K, M, S, W. Z, iso.). Jonkoa, 20-12-1899. $1150 \mathrm{~m}$.

\section{PHAILS Lour.}

occidentalis Schltr.

374 (B, holo. †). Kubango, Kueio, 3-11-1899, I $120 \mathrm{~m}$. Reference: Cribb (1984: 281).

\section{POLYSTACHYA Hook.}

dendrobiiflora Rchb.f.

839 Type of Polystachya holochila Schltr. (B, holo. t: COI, G, K M, S. W, iso.). Lazingua, 20-4-1900, $1300 \mathrm{~m}$. Reference: Cribb (1984: 286).

\section{SATYRIUM SW}

oliganthum Schltr.

383 Type of Satyrium paludosum Rchb.f. var. parvibracteatum Schltr. (B, holo. $†$; K. W, iso.). Kubango, Kueio, 4-11-1899, I $120 \mathrm{~m}$.

\section{OROBANCHACEAE}

\section{BUCHNERA L.}

baumii Engl. \& Gilg

225 (B, holo. †; COI, G, K, M, W, Z, iso.). Kuelleis (Maramba), $5-10-1899,1400 \mathrm{~m}$

prorepens Engl. \& Gilg

875 (B. holo. †; COI, G, K, M, W, iso.). Kulei-Kutsi, 29-4-1900, $1300 \mathrm{~m}$.

strictissima Engl. \& Gilg

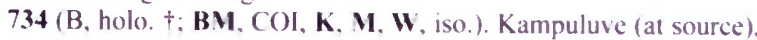
$25-2-1900,1210 \mathrm{~m}$

CYCNIUM E.Mey. ex Benth. emend. Engl.

tubulosum (L.f.) Engl. subsp. tubulosum

403 Type of Cycnium hamatum Engl. \& Gilg (B, holo. t: BM. lecto., designated by Jansen (1978: 34); COI, HBG, K. W, iso.). Quatiri (mouth), 13-11-1899.1100 m

\section{HARVEYA Hook.}

andongensis Hiern

869 Type of Harveva macramiha Engl. \& Gilg (B. holo. \$: COI. E. K. M. W, iso. ). Kulei, 26-4-1900, $1300 \mathrm{~m}$.

\section{SOPUBIA Buch.-Ham. ex D.Don}

\section{mannii Skan}

475 Type of Sopubia dregeana Benth. var. temifolia Engl. \& Gilg (B. holo, +: COI, E, G, K. M. W, iso.). Habungu, Maramba, 28-11$1899.1100 \mathrm{~m}$

\section{PASSIFLORACEAE}

\section{BASANANTHE Peyr}

baumii (Harms) W.J.de Wilde

391 Type of Tryphostemma haumii Harms (B. holo. t?: *BM. ${ }^{*} \mathrm{CO}$, G, ${ }^{*} \mathrm{~K}$, M, W. Z. iso.). Kubango (right bank), Kueio (right bank), 7-11-1899, I 120 m

\section{PEDALIACEAE}

\section{SESA MUM L.}

calycinum Welw. suhsp. repens (Fngl. \& (jilg) Seidenst. 660) Type of Sesamum repens Engl. \& (iilg (B. holo, +; K. W. iso, ) L.onga (left bank), Lazingua, 20-1-19(0), $1250 \mathrm{~m}$.

\section{POACEAE}

\section{ELYTROPHORUS P.BLaU:}

globularis Hack

112 Type of Elytrophorus interruptus Pilg. (B. holo. †'?; BW, K. M. W. iso.). Kunene (left bank). Soba (iongo (near). 17-9-1899. I $115 \mathrm{~m}$.
SPOROBOLUS R.Br.

welwitschii Rendle

511 Type of Sporobolus haumianus Pilg. (B, holo. +?; M, iso.). Ungombekike, 5-12-1899. I $200 \mathrm{~m}$.

\section{PODOSTEMACEAE}

\section{LEDERMANNIELLA Engl.}

warmingiana (Gilg) C.Cusser

904 Type of Sphaerothylax warmingiana Gilg (B. holo. +: BM. COI, K. M, W, Z, iso.). Kubango. Menempremp. 11-5-1900, $1300 \mathrm{~m}$.

\section{POLYGALACEAE}

\section{POLYGALA L.}

arenicola Gürke

301 (B. holo. †; BM. lecto., designated by Paiva (1998: 235); *COI, E, K. M. W, Z, iso.). Kuebe, 23-10-1899, 1170 m. Reference: Exell \& Mendonça (1937i: 107).

baumii Gürke

815 (B. holo. †: BM. lecto., designated by Paiva (1998: 231); BR ${ }^{\star}$ COI, E. K, M. W, Z. iso. ). Quiriri. Sakkemecho, 16-4-1900, $1200 \mathrm{~m}$. Reference: Exell \& Mendonça (1937i: 106).

kalaxariensis Schinz.

287 Type of Polygala kubangensis Gürke (B. holo. t: BM. *COI E. *K. M, W. iso.). Kubango, Massaca, 19-10-1899. I 150 m. References: Exell \& Mendonça (1937i: 106); Paiva (1998: 234).

nambalensis Gürke

244 (B. holo. †; BM. iso.). Nambali (Maramba), 6-10-1899, 1275 m. Reference: Exell \& Mendonça (1937i: 99).

817 Type of Polvgala psammophila Gürke. nom. illeg. (B, syn. + BM, *COI, *K, W, isosyn.). Quiriri. Sakkemecho, 16-4-1900, I 200 m. Reference: Exell \& Mendonça (1937i: 99).

871 Type of Polvgala psammophila Gürke. nom. illeg. (B. lecto. designated by Exell \& Mendonça (1937i: 99) †: BM. *COI. K. M. W, iso.). Kulei-Kutsi, 29-4-1900, $1300 \mathrm{~m}$. Note: Exell \& Mendonça (1937i) cited the two syntypes 817 and 871 . but they indicated the $B$ duplicate of the latter as type, making it a lectotype. This specimen was later destroyed.

paludicola Gürke

315a (B, syn. †). Kuebe, 23-10-1899.1170 m.

373 (B. lecto., designated by Exell \& Mendonca (1937i:100)t: BM. lecto.. designated by Paiva (1998: 209) without collection number; E. K. W. isolecto.). Kubango (right bank), Kueio (mouth), 3-11-1899, 1 120 m. Note: Exell \& Mendonça (1937i: 100) cited the three syntype collections, 373. 373a and 315a. referring to the B duplicate of the first as type. However, this duplicate was later destroyed. Paiva (1998) indicated as lectotype the duplicate at BM of a Baum collection from R.Cubango but the number was omitted. It is assumed that it is no. 373, since the other two syntype collections are from other localities.

373a (B. syn. †: BM. *COI. E. *K, W. Z, isosyn.). Longa, Maschonge, 30-12-1899. $1120 \mathrm{~m}$. Reference: Exell \& Mendonça (1937i: 10(0)

rivularis Gürke

595 (B, syn. +: *BM, *COI. K. W. Z. isosyn.). Maschonge, Napalanka, 28-12-1899. $1150 \mathrm{~m}$. Reference: Exell \& Mendonça (1937i: $105)$.

911 (BM, lecto.. designated by Paiva (1998: 224): *COI. E. K. W. Z. iso.). Kubango, Kohi. 15-5-19(0), $1350 \mathrm{~m}$. Note: Exell \& Mendonça (1937i: 105) cited the two svntvpe collections 595 and 911 . referring to the B duplicate of the latter as type. This specimen was later destroyed. The BM duplicate was later designated as lectotype by Paiva (1998).

robusta Gürke

704 Type of Polygala robusta Gürke var. coerulea Gürke (B. holo. †: BM. lecto., designated by Paiva (1998: 230): BM. BR. "COl. E. K. M. W. Z. iso.). Longa, Quiriri. 3-2-1900. $1275 \mathrm{~m}$. Reference Exell \& Mendonça (1937i: 106). Note: this is the lectotype of $P$ robusta.

704a Type of Polvgala robusfa Gürke var. rubra Gürke ( $B$, holo. t). Quiriri. Sakkemecho, 15-4-19(0), $1200 \mathrm{~m}$. References: Exell \& Mendonça (1937i: 1(66): Paiva (1948: 230). 
POLYGALA L. (cont.)

schinziana Chodat

554 Type of Polygala benguellensis Gürke (B, holo. †; BM, *COl, ${ }^{*} \mathrm{~K}$. W, Z, iso.). Jonkoa, 17-12-1899, 1150 m. References: Exell \& Mendonça (1937i: 104); Paiva (1998: 219),

\section{PRIMULACEAE}

\section{ANAGALLIS L.}

filifolia Engl. \& Gilg

906 (B, holo. †; COI, E, M, S, W, Z, iso.). Kubango, Kohi, 14-5$1900,1350 \mathrm{~m}$

\section{PROTEACEAE}

\section{FAUREA Harv.}

intermedia Engl. \& Gilg

912 (B, holo.; *BR, COI, E, K, M, iso.). Kubango, Kohi, 15-5. 1900, 1350 m. Reference: Brummitt \& Marner (1993: 7).

\section{PROTEA L.}

angolensis Welw. var. angolensis

863 Type of Protea chionantha Engl. \& Gilg var. Mpica Engl. \& Gilg (B, holo. [of $P$. chionantha]; BM. COI, E, G, K. M. Z. iso.) Chimpungu-Kulei, 25-4-1900, I 400 m.

angolensis Welw. var. divaricata (Engl. \& Gilg) Beard 920 Type of Protea chionantha Engl. \& Gilg var. divaricata Engl \& Gilg (B, holo.; BM, COI, E, G, K, M, Z, iso.). Kubango-Kassinga, 17-5-1900, I $400 \mathrm{~m}$.

baumii Engl. \& Gilg subsp. baumii 375 (B. holo.: BM. E, G. M. Z, iso.). Kubango (right bank), Kueio (mouth), 3-11-1899, I $100 \mathrm{~m}$.

gaguedi J.F.Gmel.

272 Type of Protea chrysolepis Engl. \& Gilg (B. holo.; BM, COI, E, G, M, Z, iso.). Kubango, Chirumbu, 14-10-1899, $1200 \mathrm{~m}$.

lemairei De Wild. subsp. trichophylla (Engl. \& Gilg) Beard 918 Type of Protea trichophylla Engl. \& Gilg (B, holo.; BM, COI, E. G, K. M, Z, iso.). Kubango-Kassinga, 17-5-1900, $14($ () $)$ m.

poggei Engl. subsp. haemantha Chisumpa \& Brummit 709 Type of Protea haemantha Engl. \& Gilg (B. holo:: BM, C(), E. G. K. M, Z, iso.). Longa, Quiriri, 6-2-1900, $1275 \mathrm{~m}$.

welwitschii Engl.

653 Type of Protea melliodora Engl. \& Gilg (B, holo.: BM, COI, E. G. M. Z, iso.). Longa, Minnesera, 11-1-1900, $1250 \mathrm{~m}$. 903 Type of Protea myrsinifolia Engl. \& Gilg (B. holo.; COI, G, K. M. S, Z, iso. ). Kubango, Menempremp. 8-5-190(), I $450 \mathrm{~m}$.

\section{RUBIACEAE}

\section{CALANDA K.Schum.}

rubricaulis K.Schum. var. rubricaulis 899 (B. holo. †; BR, COI, K, M, W, Z, iso.). Menempremp, Kubango (near), 14-5-1900, $1450 \mathrm{~m}$.

\section{FADOGIA Schweinf.}

chlorantha K.Schum. var. chlorantha 482 ( B, holo. †; E, W. Z, iso.). Habungu, 28-11-1899, I $100 \mathrm{~m}$.

chlorantha K.Schum. var. thamnus (K.Schum.) Verdc. 491 Type of Fadogia thamnus K.Schum. (B. holo. †: BV. E. K. M. S. W, Z, iso.). Habungu, 22-11-1899, 1 100 m.

\section{chrysantha K.Schum.}

210 (B, holo.; BM. M, S, W, Z, iso.). Kassinga, 4-1(0-1899, $1290 \mathrm{~m}$.

\section{GARDENIA Ellis}

brachythamnus (K.Schum.) Launen

389 Type of Randia brachythamnus K.Schum. (B. holo. +: BM COI. M. W, Z, iso.). Kubango (right bank), Kueio, 5-11-1894, $1100 \mathrm{~m}$.
LEPTACTINA Hook.i

pretrophylax K.Schum.

137 (B, holo. †; BM. E, G, W, Z. iso.). Chitanda (left bank), 23-9_ 1899, $1105 \mathrm{~m}$.

prostrata K.Schum

713 (B. holo. †; E, G, W, iso.). Quiriri, Sakkemecho, 13-2-1900, I $300 \mathrm{~m}$.

\section{NEOPENTANISIA Verdc.}

annua (K.Schum.) Verdc.

659 Type of Pentanisia annua K.Schum. (B, holo. †; BM. K. M. W, Z, iso.). Longa (left bank), Lazingua, 20-1-1900, $1250 \mathrm{~m}$.

\section{PAVETTA L}

arenicola K.Schum

522 (B. holo. †; E. W, iso.). Ungombekike Kuito, 10-12-1899, $1200 \mathrm{~m}$.

paupercula K.Schum.

805 (B, holo, †; BM. E, K. W. Z, iso.). Quiriri, Sakkemecho, 9-4$1900,1200 \mathrm{~m}$

stipulopallium K.Schum.

948 (B, holo.; BM, E, K. M, W, Z, iso.). Chitanda (left bank), 28$5-1900,1150 \mathrm{~m}$.

\section{RYTIGYNIA Blume}

orbicularis (K.Schum.) Robyns

581 Type of Plectronia orhicularis K.Schum. (B. holo; BM, E, K.

M. W, Z, iso.). Longa, Napalanka, 25-12-1899, 1 I50 m

VANGUERIA Juss.

proschii Briq.

446 Type of Vangueria lasioclados K.Schum. (B, holo,; C(), E. K. M, W. Z, iso.). Kubango (left bank), Kalolo, 20-11-1899.

\section{SANTALACEAE}

\section{THESIU M L.}

leucanthum Gilg

218 (B, holo. †?; COI, E. K. W, iso.). Kassinga-Kolove, 4-10$1899, \mathrm{I} 300 \mathrm{~m}$.

lycopodioides Gilg

879 (B, holo. †"? E. K. W. iso.). Kulei-Kutsi, 29-4-1900, $1300 \mathrm{~m}$.

\section{SCROPHULARIACEAE}

\section{BAUMIA Engl. \& Gilg}

angolensis Engl. \& Gilg

785 (B, holo. t?: S. iso.). (Onschingue, Kuito (ncar), 29-3-1900, $1200 \mathrm{~m}$.

\section{DOPATRIUM Benth.}

stachytarphetoides Engl. \& Gilg 923 (B, holo. †?; COI, E, M, S, W, iso.). Kubango-Kassinga, $17-$ $5-1900,1400 \mathrm{~m}$.

\section{L.IMNOPHILA R.Br.}

ceratophylloides (Hiern) Skan

750 Type of Ambulia haumii Engl. \& Gilg (B. holo. +?; COI. K. M S. W, Z, iso.). Kutue, 2-3-19(0), 1 200 $\mathrm{m}$.

dasyantha (Engl. \& Gilg) Skan

729 Type of Ambulia dasvantha Engl. \& Gilg (B, holo, t?; COI, K I. S. W. iso. ). Quiriri, Sobi, 2-19(K), $1285 \mathrm{~m}$.

\section{SIMAROUBACEAE}

\section{KIRKIA Oliv.}

acuminata ()liv

966 p.p. quoad tructus. Type of Kirkio glanca Engl. \& (iilg (B. holo. +?). Makopi-Gambos, 8-6-19(0), $1200 \mathrm{~m}$. Reference: Exell \& Mendonça (1951e: 276). 


\section{QUASSIA L.}

undulata (Guill. \& Perr.) D.Dietr.

674 Type of Hannoa chlorantha Engl. \& Gilg (B. holo. †: *BM, COI, K, M, S, W, iso.). Longa, Lazingua, 23-1-1900, $1250 \mathrm{~m}$. References: Exell \& Mendonça (1951e: 278); Stannard (2000: 11).

\section{STERCULIACEAE}

\section{HERMANNIA L.}

eenii Baker $f$.

514 Type of Hermannia angolensis K.Schum. (B, holo. +?; *BM.

COI, E, G, K. M, W, Z, iso.). Ungombekike-Kuito, 9-12-1899,

I 200 m. Reference: Exell \& Mendonça (195 ]f: 195).

\section{STRYCHNACEAE}

\section{STRYCHNOS L.}

cocculoides Baker

290 Type of Strychnos schumanniana Gilg (B. holo. †: BM. E. K.

W, Z, iso.). Kubango, Massaca, 19-10-1899. I 150 m.

\section{THYMELAEACEAE}

\section{GNIDIA L.}

baumiana Gilg

211 (B, holo. †; COI, M, W, iso.). Chitanda, Kassinga, 4-10-1899.

$1290 \mathrm{~m}$.

pleurocephala Gilg

828 (B, holo. †; W, iso.). Longa. 18-4-1900, 1275 m.

\section{TILIACEAE}

GREWIA L.

avellana Hiern

289 Type of Grewia perennans K.Schum. (B. holo. t; G. iso.) Kubango, Massaca, 19-10-1899, 1 150 m. Reference: Exell \& Mendonça (1951g: 218).

535 Type of Grewia hidrophila K.Schum. (B. holo. †: *BM, COI

E. G. ${ }^{*}$ K, M, Z, iso.). Kuito (near), 12-12-1899. Reference: Exell \& Mendonça (1951g: 218).

\section{falcistipula K.Schum.}

353 (B, holo. †; COI, E, G, K. M, S, W. Z, iso.). Kubango (right bank), Kueio, 1-11-1899, $1300 \mathrm{~m}$. Reference: Exell \& Mendonça (1951g: 216).

schinzii K.Schum.

420 Type of Grewia pinacostigma K.Schum. (B. holo. †: BM. E. G, W, iso.). Kubango (left hank), Kavanga. 18-11-1899. 1 100 m Reference: Exell \& Mendonça (1951g: 217).

suffruticosa K.Schum.

189 Type of Grewia brevicaulis K.Schum. (B. holo. †: *BM. COI. E. G, ${ }^{*}$ K. M, S, Z. iso.). (joudkopje-Kakele, 3-10-1899, $1210 \mathrm{~m}$. Reference: Exell \& Mendonça (1951g: 224).

\section{VERBENACEAE}

\section{I.IPPIA I}

baumii Gürke var. haumii 515 (B, holo, t: ${ }^{*} B \mathrm{M}$, COI, G, K, M, W, iso.). UngombekikeKuito, 9-12-1899, $1200 \mathrm{~m}$. Reference: Verdcoun (1992: 32).

\section{VITACEAE}

\section{AMPLOCISSUS Planch.}

\section{brunneo-rubra Gilg}

509 (B, holo, +: "BM, COI, G, K. W, iso.). Ungombekike, 5-12$1899.1200 \mathrm{~m}$. Reference: Exell \& Mendonça (1954c: 38).

\section{CISSUS L.}

jatrophoides ( Welw. ex Baker) Planch 278 Type of (issus chloromiha Gilg (B. holo, t: (, W. 1so), Kubango, Chirumbu, 14-10-1899, 1200 m.

\section{CYPHOSTEMMA (Planch) Alston}

chloroleucum (Welw. ex Baker) Desc. ex Wild \& R.B.Drumm. 361 Type of Cissus hypargirea Gilg (B, holo. +: *BM. COI. C. ${ }^{*} \mathrm{~K}$. W. iso.). Kubango (left bank), Kabindere, 2-11-1899, $1150 \mathrm{~m}$. Reference: Exell \& Mendonça (1954c: 60).

fugosioides (Gilg) Desc. ex Wild \& R.B.Drumm. 174 (B. holo. †; *BM. COI. G. ${ }^{*}$ K. M. W. iso.). Chitanda (right margin), 27-9-1899. $1150 \mathrm{~m}$. Reference: Exell \& Mendonça (1954c: 58).

violaceo-glandulosum (Gilg) Desc

270 (B. lecto., designated by Exell \& Mendonça (1954c: 56)t; *BM, COI, G, *K, M. S, isolecto.). Kubango, Chirumbu, 11-101899. $1200 \mathrm{~m}$. Note: Exell \& Mendonça (1954c) cited the two syntypes but only 270 is referred to as 'type'. This is considered a lectotypification.

276a (B, syn. †). Kubango, Chirumbu, 10-1899. I $200 \mathrm{~m}$. Reference: Exell \& Mendonça (1954c: 56).

\section{XYRIDACEAE}

\section{XYRIS L}

congensis Büttner

333 Type of Xiris haumii L.A.Nilsson (B, holo.; BM. BR. K. M iso.). Kuebe and Kubango (confl.). 28-10-1899, $1150 \mathrm{~m}$.

\section{ACKNOWLEDGEMENTS}

The authors would like to thank: Ms Hester Steyn and Ms Sandra Turck, SANBI, for the map; Dr Eurico Martins, for information on collecting localities; Dr David Goyder and Dr Henk Beentje, for information on types.

\section{REFERENCES}

BANCROFT. H. 1937. Dipterocarpaceae. Conspectus florae angolensis 1: 132-142. Junta de Investigações do Ultramar, Lisboa.

BRETELER, F.J. 1978. The African Dichapetalaceae IV. Mededelingen Landbouwhogeschool Wageningen 78-10: 1-84.

BRU MMITT, R.K. \& MARNER. S.K. 1993. Proteaceae. Flora of tropical East Africa. Balkema, Rotterdam.

CANNON, J.F.M. 1970. Umbelliferae. Conspectus florae angolensis 4 334-360. Junta de Investigações do Ultramar and Instituto de Investigação Cientifica de Angola, Lisboa.

COWLEY, E.J. 1988. Burmanniaceae. Flora of tropical East Africa. Balkema. Rotterdam.

CRIBB, P.J. 1984. Orchidaceac. Part 2. Flora of Iropical East Africa. Balkema, Rotterdam.

CRIBB. P.J. 1989. Orchidaceac, Pan 3. Flora of tropical East Africa. Balkema, Rotwerdam.

EXELL. A.W. \& GARCIA, J. 1970. Combretaceac. Conspecrus flora angolensis 4: 44-93. Junta de Investigaçôes do Ultramar and Instituto de Investigação Cientifica de Angola. Lisboa.

EXELL, A.W. \& MENDONCA. F.A. 1937a. Cruciferac. Conspectus force angolensis 1: 49-52. Junta de Investigaçōes do Lltramar. Lisboa.

EXELL, A.W \& MENIONCA. F.A. 1937b. Capparidaceac. Conspecuus fiorce angolensis 1: 53-68. Junta de Investigaçôes do LItramar, Lisbua.

EXELL, A.W. \& MENDONCA. F.A. 1937c. Caryophyllaceae. Conspectus florcle angolensis 1: 108-113. Junta de Investigacoies do Ultramar, Lisboa.

EXELL, A.W. \& MENIONCA. F.A. 1937d. Elatinaceac. Conspecrus florae angolensis 1: 118. 119. Junta de Investigaçôes do Ultramar, Lisbova.

EXELL. A.W. \& MENIXONC A. F.A. 1937e. Hypericaceac. Conspectus florae angolensis I: 119-124. Junta de Investigaçóes do Litramar, Listua.

EXELL. A.W. \& MENIXON(A. F.A. 1937f. Malvaceae. Conspectus florce angolensis 1: 146 176. Junta de Investigaciés do Ultramar, Lishora. 
EXELL, A.W. \& MENDONÇA, F.A. 1937g. Menispermaceae. Conspectus florae angolensis 1: 32-45. Junta de Investigaçòes do Ultramar, Lisboa.

EXELL, A.W. \& MENDONÇA, F.A. 1937h. Nymphaeaceae. Conspectus florae angolensis 1: 45-48. Junta de Investigações do Ultramar, Lisboa.

EXELL, A.W. \& MENDONÇA, F.A. 1937i. Polygalaceac. Conspecrus florae angolensis 1: 87-108. Junta de Investigações do Ultramar, Lisboa.

EXELL. A.W. \& MENDONCA. F.A. 1951a. Ixonanthaceae. Conspectus florae angolensis 1: 246-248. Junta de Investigações do Ultramar, Lisboa.

EXELL, A.W. \& MENDONÇA, F.A. 1951b. Malpighiaceae. Conspectus florae angolensis 1: 250-254. Junta de Investigações do Ultramar, Lisboa.

EXELL, A.W. \& MENDONCA, F.A. 195lc. Meliaceae. Conspectus florae angolensis 1: 305-320. Junta de Investigaçôes do Ultramar, Lisboa.

EXELL, A.W. \& MENDONCA, F.A. 195Id. Ochnaceae. Conspectus florae angolensis 1: 283-297. Junta de Investigaçōes do Ultramar, Lisboa.

EXELL, A.W. \& MENDONÇA, F.A. 1951e. Simaroubaceae. Conspectus florae angolensis 1: 276-279. Junta de Investigações do Ultramar, Lisboa.

EXELL, A.W. \& MENDONÇA, F.A. 195If. Sterculiaceae. Conspectus florae angolensis 1: 184-208. Junta de Investigaçōes do Ultramar, Lisboa.

EXELL, A.W. \& MENDONCA. F.A. 1951 g. Tiliaceac. Conspectus florae angolensis 1: 208-241. Junta de Investigações do Ultramar, Lisboa.

EXELL. A.W. \& MENDONÇA, F.A. 1954a. Anacardiaceae. Conspectus florae angolensis 2: 95-134. Junta de Investigaçòes do Ultramar, Lisboa.

EXELL, A.W. \& MENDONÇA, F.A. 1954b. Celastraceae. Conspectus florce angolensis 2: 1-27. Junta de Investigações do Lltramar, Lisboa.

EXELL. A.W. \& MENDONÇA, F.A. 1954c. Vitaceac. Conspectus florae angolensis 2: 35-71. Junta de Investigações do Lltramar, Lisboa.

FAYED, A. 1979. Revision der Grangeinae (Asteraceae-Astereae). Mitteilungen der Botanischen Staatssammlung München 15: $425-576$.

FERNANDES, R. 1966. Estudo das Anacardiaceae africanas. I. Contribuição para o conhecimento do género Ozoroa Del. Garcia de Orta 14,1: 19-60.

FERNANDES, R. \& FERNANDES, A. 1970. Onagraceae. Conspectus florae angolensis 4: 190-203. Junta de Investigações do Ultramar and Instituto de Investigação Cientifica de Angola, Lisboa.

FIGUEIREDO, E. \& SMITH, G.F. 2008. Plants of Angola-Plantas de Angola. Strelitzia 22. South African National Biodiversity Institute, Pretoria.

GEBAUER. R. 1993. The type specimens of African Loranthus taxa (Loranthaceae) described by Engler and kept in the general herharium at Berlin-Dahlem (B). Willdenowia 23: 257-289.

HEINTZE, B. 2007. Deutsche Forschungsreisende in Angola-Ethnografische Aneignungen. Verlag Otto Lembeck. Frankfurt-Main.

HIEPKO, P. 1987. The collections of the Botanical Museum BerlinDahlem (B) and their history. Englera 7: 219-252

HILLCOAT, D. 1966. Sophoreae. Conspectus florue angolensis 3,2: 372-384. Junta de Investigações do Lltramar, Lishoa.

HOLMGREN, P.K., HOLMGREN. N.H. \& BARNETT. L.C. 1990. Index herhariorum. Part I: The Herbaria of the World. New York Botanical Garden. Bronx, New York

JANSEN, O.J. 1978. The genus Cicnium Benth. emend. Engl. (Scrophulariaceae). A taxonomic revision. Dansk Botanisk Arki. 32,3: $1-72$.

KOKWARO, J.O. 1986. Anacardiaceae. Flora of tropical East Africa. Balkema. Rotterdam.

LANJOUW, J. \& STAFLEU, F.A. 1954. Index Herbariorum. Part II. Collectors A-D. Regnum legetabile 2: 1-174. International Bureau for Plant Taxonomy and Nomenclature, Utrecht.

MANSFELD, P. 1983. Hugo Baum, eine Biographie. http:/baum.petermansfeld.de.
MENDES, E. 1970. Anisophyllea. Conspectus florae angolensis 4: 4044. Junta de Investigaçoes do Ultramar and Instituto de Investigação Científica de Angola. Lisboa.

MENDONCA, F.A. 1943. Contribuicões para o conhecimento da flora de Angola. I. Compositae. Junta das Missões Geográficas e de Investigaçòes Coloniais, Lisboa.

MILNE-REDHEAD, E. 1953. Hypericaceae. Flora of tropical East Africa. Balkema, Rotterdam.

NEMOMISSA, S. 2002. Gentianaceae. Flora of tropical East Africa. Balkema. Rotterdam.

PAIVA, J. 1998. Polygalarum Africanarum et Madagascariensium Prodromus atque gerontogaei generis Heterosamara Kuntze, a genere Polygala L. segregati et a nobis denuo recepti, synopsis monographica. Fontqueria 50: 1-346.

PERSOON, J.G.M., VAN DILST, F.J.H., KUIJPERS, R.P., LEEUWENBERG, A.J.M. \& VONK. G.J.A. (eds). 1992. The African species of Landolphia P. Beauv. Wageningen Agricultural University Papers 92-2: 1-250.

RAYNER. T.G.J. 1992. Studies on the genus Bidens L. (Compositae) from the eastern hemisphere. 3. Typification of names of Bidens, Coreopsis L., Guizotia Cass., and Microlecane (Sch.Bip.) Benth. \& Hook.f. from Africa. Phytologia 73: 77-97.

SLEUMER, H. 1937. Flacourtiaceac. Conspectus florcue angolensis 1: 78-87. Junta de Investigaçòes do Ultramar, Lisboa.

SMITH, C.A. 1966. Common names of South African plants. Memoirs of the Botanical Survey of South Africa No. 35

SOUSA. E.P. 1962. Seshania. Conspectus forae angolensis 3,1: 180186. Junta de Investigaçồes do Ultramar, Lisboa.

STANNARD, B. 2000. Simaroubaceac. Flora of tropical East Africa. Balkema, Rotterdam.

SUMMERHAYES, V.S. 1968. Orchidaceae, Part 1. Flora of tropical East Africa. Balkema, Rotterdam.

TAYLOR, P. 1973. Lentibulariaceac. Flora of tropical East Africa. Balkema, Rotterdam.

THULIN, M. 1975. The genus Wahlenbergia s.lat. (Campanulaceae) in tropical Africa and Madagascar. Simbolae Botanicae Upsalienses 21: 1-223.

THULIN, M. 1979. Monopsis (Loheliaceae) in tropical Africa. Botaniska Notiser 132: 131-137.

THULIN, M. 1984. Lobeliaceac. Flora of tropical East Africa. Balkema, Rotterdam.

TORRE, A.R. 1962. Papilionoideae: Genisteae-Galegeac. Conspecrus forcae angolensis 3,1: 1-187. Junta de Investigaçòes do Ultramar, Lisboa.

TORRE, A.R. 1965. Taxa angolensia nova vel minus cognita-IV. Boletim da Sociedade Broteriana, sér. 2, 39: 207-229.

TORRE, A.R. 1966. Papilionoideac: Hedysareae-Sophoreac. ConspecIus florae angolensis 3,2:188-401.

TORRE, A.R. \& HILlCOAT, D. 1956. Caesalpinioideae. Conspectus florae angolensis 2,2: 162-253. Junta de Investigaçōes do Ultramar, Lisboa.

TOWNSEND, C.C. 1989. Umbelliferae. Flora of tropical East Africa. Balkema. Rotterdam.

VENTER, H.J.T., VERHOEVEN, R.L. \& KOTZE, J.D.S. 1990. A monograph of Tacazzea (Periplocaceac). South African Journal of Borany 56: 93-112.

VERI)COURT. B. 1984. Ixonanthaceae. Flora of tropical East Africa. Balkema, Rotterdam.

VERDCOURT, B. 1992. Verhenaceae. Flora of tropical East Africa. Balkema, Rotterdam.

VERICCOURT, B. 2(K)5. Ochnaceac. Flora of iropical East Africa. Royal Botanic (jardens, Kew

WARBURG, O. 19013. Kunene-Sambesi-Expedition. H. Baum. Kolonial-Winschaftliches Komitec. Berlin.

Website addresses:

Aluka: www.aluka.org

Berlin Herbarium (B): www.bgbm.org

http://ww.bgbm.org/BGBM/research/colls/herb/phanerog.htm List of families including extant collections of B before 1943 (accessed 2007).

Kew Herbarium (K): www.kew.org/herbcat

Zurich Herbarium $(Z)$ : ww w.zuerich-herbarien.unizh.ch 


\section{INDEX TO PLANT NAMES}

Note: synonyms in italics, accepted names in bold.

Adenodolichos

baumii Harms, 195

euryphillus Harms, 195

rhomboideus (O.Hoffm.) Harms var. rhomboideus. 195

Aeollanthus

engleri Briq., 197

paludosus Gürke, 197

uliginosus Gürke, 197

leschynomene

baumii Harms, 195

bracteosa Welw. ex Baker, 195

nambalensis Harms, 195

Agelanthus terminaliae (Engl. \& Gilg) Polhill \& Wiens, 197 Aloe

haumii Engl. \& Gilg, 192

brunneo-punctala Engl. \& (iilg, 192

metallica Engl. \& Gilg, 192

nuttii Baker, 192

zebrina Baker, 192

Ambulia

baumii Engl. \& Gilg, 200

dasvantha Engl. \& Gilg, 200

Ammocharis baumii (Harms) Milne-Redh. \& Schweick., 191

Ampelocissus hrunneo-rubra Gilg, 201

Amphidoxa lasiocephala O.Hoffm., 192

Anagallis filifolia Engl. \& Gilg, 200

Aneilema plagiocapsa K.Schum., 194

\section{Anisopappus}

chinensis Hook. \& Arn. subsp. chinensis var. chinensis, 192 gracilis O.Hoffm., 192

subdiscoideus O.Hoffm., 192

Anisophyllea

fruticulosa Engl. \& Gilg, 191

quangensis Engl, ex Henriq.. 191

\section{Anthericum}

liliagastrum Engl. \& Gilg, 191

pallidiflarum Engl. \& Gilg, 192

xylorrhizum Engl. \& Gilg, 192

Aniholyza magnifica Harms, 197

Artemisiopsis villosa (O.Hoffm.) Schweick., 192

Asclepias

baumii Schltr. 192

leucotricha Schltr., 192

Asparagus

altiscandens Engl. \& Gilg. 192

baumii Engl. \& Gilg, 192

Aspidoglossum masaicum (N.E.Br.) Kupicha. 192

Baikiaea plurijuga Harms, 195

Baphia

cornifolia Harms, 195

massaiensis Taub. subsp. obovata (Schinz) Brummitt var. cornifolia (Harms) Soladoye. 195

Basananthe baumii (Harms) W.J.de Wilde. 199

Baumia angolensis Engl. \& (iilg. 200)

Belmonica

haumiana (iilg. 196

chionamho (jilg. 196

Benguellia lanceolata (Gürke) (j. Taylor, 197

\section{Bergia}

enthrolenca (jilg, 195

pullide-rosea Ciilg. 195

pentheriana Keissl. 195

spathulata Schinz. 195

Berlinia baumii Harms. 196

Bidens

baumii (O.Hoftm.) Sherli, 192

flabellata O.Hoftim., 192

Boortic

aschersoniana Gürke. 196

kunenensis Gürke, 196

Boscia

suaveolens Gilg. 194

nelwitschii (iilg. 194

Bothriocline linearifolia O.Hoffin. 193

\section{Brachycorythis}

angolensis (Schltr.) Schltr., 198

oligophylla Kraenzl., 198

rhodostachỵs (Schltr.) Summerh., 198

Buchnera

baumii Engl. \& Gilg. 199

prorepens Engl. \& Gilg. 199

strictissima Engl. \& Gilg. 199

Bulbine

abyssinica A.Rich. 192

xamthobotrys Engl. \& Gilg. 192

Burmannio

hicolor Mart. var. micrantha Engl. \& Gilg. 193

blanda Engl. \& Gilg. 193

madagascariensis Mart. 193

Calanda rubricaulis K.Schum. var. rubricaulis, 200

Carpodinus leucantha K.Schum.. 192

Cephalaria retrosetosa Engl. \& Gilg. 195

Chironia baumiana Gilg, 196

Chlorophytum

colubrinum (Baker) Engl., 192

dolichostachys Engl. \& Gilg. 192

psammophilum Engl. \& Gilg. 192

Cissus

chlorantha Gilg, 201

fugosioides Gilg. 201

hipargirea Gilg. 201

jatrophoides (Welw. ex Baker) Planch., 201

Clerodendrum baumii Gürke. 197

Coleus haumii Gürke, 197

Combretum

arhuscula Engl. \& Gilg. 194

arenarium Engl. \& Gilg. 194

angirochroum Engl. \& Gilg. 194

aureonitens Engl. \& Gilg. 194

haumii Engl. \& Gilg. 194

collinum Fresen., 194

gnidioides Engl. \& Gilg. 194

laeteviride Engl. \& Gilg. 194

molle R.Br. ex G.Don. 194

monricola Engl. \& Gilg. 194

pachicarpum Engl. \& Gilg, 194

platypetalum Welw. ex M.A.Lawson subsp. baumii (Engl. \& Gilg)

Exell, 194

psidioides Welw. subsp. dinteri (Schinz) Exell. 194

quirirense Engl. \& Gilg. 194

Commelina

elegantula K.Schum., 194

filifolia K.Schum.. 194

milne-redheadii Faden. 194

subulata Roth. 194

welw itschii C.B.Clarke. 194

welwitschii C.B.Clarke var. glahra K.Schum., 194

Conyza

limosa O.Hoffm., 192

welwitschii (S.Moore) Wild. 192

Copaifera baumiana Harms. 195

Coreopsis buumii O.Hoffm.. 192

Crassocephalum uvens (Hiern) S.Moore, 192

Crinum baumii Harms, 191

Crotalaria

angulicaulis Harms. 195

haumii Harms, 195

densicephala Welw. ex Baker, 195

lachnoclada Harms, 195

leptoclada Harms var. leptoclada, 195

psammophila Harms. 195

sericifolia Harms. 195

subsessilis Harms. 195

Cryptolepis

haumii Schltr., 192

producta N.E.Br., 192

Cycnium

hamarum Engl. \& Gilg. 194

tuhulosum (L.f.) Engl. subsp. tuhulosum. 194 
Cyperus chrysocephalus (K.Schum.) Kük., 195

Cyphostemma

chloroleucum (Welw. ex Baker) Desc. ex Wild \& R.B.Drumm., 201

fugosioides (Gilg) Desc. ex Wild \& R.B.Drumm., 201

violaceo-glandulosum (Gilg) Desc., 201

Desmonema pallide-aurantiacum Engl. \& Gilg, 198

Dichapetalum

cymosum (Hook.) Engl., 195

venenarum Engl. \& Gilg, 195

Dicoma

anomala Sond., 193

subsp. anomala, 193

var. latifolia O.Hoffm. 193

var. microphylla O.Hoffm., 193

antunesii O.Hoffm., 193

Diospyros

batocana Hiern, 195

baumii Gürke, 195

kirkii Hiem, 195

virgata (Gürke) Brenan, 195

xanthocarpa Gürke, 195

Dipcadi

anthericoides Engl. \& Gilg, 196

baumii Engl. \& Gilg, 196

lividescens Engl. \& Gilg, 196

Iongifolium (Lindl.) Baker. 196

vaginatum Baker, 196

viride (L.) Moench, 196

Diplolophium zambesianum Hiern, 192

Dopatrium stachytarphetoides Engl. \& Gilg, 200)

Duosperma

quadrangulare (Klotzsch) Brummitt. 191

sessilifolium (Lindau) Brammitt, 191

Elytrophorus

globularis Hack., 199

interruptus Pilg., 199

Emilia

baumii (O.Hoffm.) S.Moore, 193

limosa (O.Hoffm.) C.Jeffrey, 193

Entada

arenaria Schinz subsp. arenaria. 195

nana Harms, 195

Entandrophragma ekebergioides (Harms) Sprague, 198

Erigeron baumii O.Hoffm., 193

Erlangea linearifolia (O.Hoffm.) S.Moore, 193

Erythrina baumii Harms, 196

Euclea

baumii Gürke, 195

crispa (Thunb.) Gürke, 195

divinorum Hiem. 195

huillensis Gürke, 195

Eulophia

adenoglossa (Lindl.) Rchb.f., 198

arenicola Schltr., 198

haumiana Kraenzl., 198

calantha Schltr., 198

calantha Schltr. var. kubangensis Schltr., 198

coeloglossa Schltr., 198

corymbosa Schltr., 198

gonychila Schltr., 198

macra Schltr., 198

malangana (Rchb.f.) Summerh., 198

rolfeana Kraenzl., 198

tricristata Schltr., 198

trilamellata De Wild., 198

warhurgii Schltr., 198

Fadogia

chlorantha K.Schum., 20()

var. chlorantha. 200

var. thamnus (K.Schum.) Verdc., 200

chrysantha K.Schum., 20()

thamnus K.Schum., 200)

Faurea intermedia Engl. \& Gilg. 200)

Floscopa

Havida C.B.Clarke, 194

pusilla K.Schum., 194

Gardenia brachythamnus (K.Schum.) Launert. 200 )

Geniosporum haumii Gürke, 197
Gladiolus

haumii Harms, 197

benguellensis Baker, 197

kubangensis Harms, 197

longanus Harms, 197

magnificus (Harms) Goldblatt, 197

pallidus Baker, 197

Gnidia

baumiana Gilg, 20

pleurocephala Gilg, 201

Grangea anthemoides O.Hoffm., 193

Grewia

avellana Hiern, 201

brevicaulis K.Schum., 201

falcistipula K.Schum., 201

hidrophila K.Schum., 201

perennans K.Schum., 201

pinacostigma K.Schum., 201

schinzii K.Schum., 201

suffruticosa K.Schum., 201

Gimnema melananthum K.Schum., 192

Gymnosporia

baumii Loes., 194

senegalensis (Lam.) Loes., 194

Habenaria

holothrix Schltr., 198

holubii Rolfe, 198

huillensis Rchb.f., 198

kuhangensis Schltr., 198

macroplectron Schltr., 198

monophvlla Schltr., 199

rhopaloceras Schltr., 198

verdickii (De Wild.) Schltr. 199

Hannoa chlorantha Engl. \& Gilg, 201

Harveva

andongensis Hiem, 199

macrantha Engl. \& Gilg. 199

Heeria

anglrochrysea Engl. \& Gilg. 191

benguellensis Engl. var. petrophila Engl. \& Gilg, 191

longipes Engl. \& Gilg. 191

stenophylla Engl. \& Gilg, 191

.rylophylla Engl. \& Gilg, 19]

Helichrysum congolanum Schltr. \& O.Hoffm., 193

Hermannia

angolensis K.Schum., 201

eenii Baker f., 201

Hibiscus baumii Gürke, 197

Hydrostachys triaxialis Engl. \& Gilg. 197

Higrophila

affinis Lindau, 191

glutinifolia Lindau. 191

sessilifolia Lindau, 191

Hypericum

baumii Engl. \& Gilg. 197

lalandii Choisy, 197

Hvpris hatumii Gürke, 197

Indigofera

baumiana Harms, 196

griseoides Harms, 196

namhalensis Harms, 196

Inula

limosa O.Hoffm., 193

paludosa (O.Hoffm. 193

Ipomoea

crassipes Hook., 194

var. hirla Hallier f. 194

Jatropha haumii Pax. 195

Julbernardia paniculata (Benth.) Troupin, 196

Kalanchoe

haumii Engl. \& Gilg. 194

brachyloba Welw. ex Britten, 194

Kirkia

acuminata Oliv., 20)

glauca Engl. \& Gilg. 200)

Laggera humilis ().Hoffm., 193

L.andolphia camptoloba (K.Schum.) Pichon, 192

Ledebouria revoluta (L.f.) Jessop. 196

Ledermanniella warmingiana (Gilg) C.Cusset. 199

Lepidagathis macrochila Lindau. 191 
Leptactina

pretrophylax K.Schum., 200

prostrata K.Schum., 200

Lightfootia laricifolia Engl. \& Gilg, 194

Limnophila

ceratophylloides (Hiern) Skan, 200)

dasyantha (Engl. \& Gilg) Skan. 200

Lippia baumii Gürke var. baumii. 20|

I obelia

angolensis Engl. \& Diels ex Diels, 197

fonticola Engl. \& Gilg, 197

minutidentata Engl. \& Gilg, 197

Loranthus

haumii Engl. \& Gilg. 197

terminaliae Engl. \& Gilg, 197

Ludwigia

pulvinaris Gilg. 198

senegalensis (DC.) J.Troch., 198

Maha virgata Gürke, 195

Mariscus chrysocephalus K.Schum., 195

Melanthera

haumii O.Hoffm. 193

triternata (Klatt) Wild, 193

Monopsis zevheri (Sond.) Thulin. 197

Monotes dasyanthus (iilg, 195

Nasturtium microcapsum Engl. \& Gilg, 193

Neopentanisia annua (K.Schum.) Verdc., 200

Nesaea baumii Koehne, 197

Neurotheca

haumii Gilg, 196

congolana De Wild. \& T.Durand, 196

Nicolasia felicioides S.Moore, 193

Nidorella

densiftora ().Hoffm., 193

linearifolia O.Hoffm., 192

resedifolia DC. subsp. resedifolia. 193

Nymphaea sulphurea (iilg. 198

Ochna

annunesii Engl. \& Gilg, 198

arenaria De Wild. \& T.Durand, 198

brunnescens Engl. \& Gilg, 198

cinnabarina Engl. \& Gilg, 198

hoepfneri Engl. \& Gilg. 198

pulchra Hook., 198

pygmaea Hiern, 198

roseiflora Engl. \& Gilg. 198

Oncoba longipes Gilg, 196

Orthosiphon lanceolanus Gürke, 197

\section{Ottelia}

hanmii Gürke, 196

benguellensis Gürke, 196

kunenensis (Gürke) Dandy, 196

muricata (C.H.Wright) Dandy, 196

ulvifolia (Planch.) Walp., 196

Ozoroa

argy rochrysea (Engl. \& (iilg) R.Fern. \& A.Fem.. 191

longipes (Engl. \& (jilg) R.Fern. \& A.Fern. 191

stenophylla (Engl. \& Gilg) R.Fern. \& A.Fern., 191

xylophylla (Engl. \& (iilg) R.Fern. \& A.Fern. 191

Pasaccardoa baumii O.Hoffm., 193

Pavetta

arenicola K.Schum. 200

paupercula K.Schum., 200

stipulopallium K.Schum., 200

Pavonia senegalensis (Cav.) Leistner. 197

Pentanisia anmura K.Schum., 200

Phaius occidentalis Schltr., 199

Phragmanthera baumii (Engl. \& (iilg) Polhill \& Wiens, 197

Phyllocosmus

candidus Engl. \& Gilg. 197

Iemaireanus (DE Wild. \& T.Durand) T.Durand \& H.Durand, 197

Physostigma

mesoponticum Tauh. 196

var. banmii Harms, 196

Physorrichia arenaria Engl. \& Gilg. 192

Platamthera

angolensis Schltr., 198

rhodestachys Schler. 198

Platostoma strictum (Hiem) A.J.Paton, 197
Plectranthus

baumii Gürke, 197

betonicifolius Baker, 197

guerkei Briq., 197

Plecrronia orbicularis K.Schum. 200

Polycarpaea inaequalifolia Engl. \& Gilg. 194

Polygala

arenicola Gürke, 199

baumii Gürke, 199

bengwellensis Gürke, 200

kalaxariensis Schinz, 199

kubangensis Gürke, 199

nambalensis Gürke, 199

paludicola Gürke. 199

psammophila Gürke, 199

rivularis Gürke, 199

robusta Gürke, 199

var. coerulea Gürke. 199

var. rubra Gürke, 199

schinziana Chodat. 200

Polystachva

dendrobiiflora Rchb.f., 199

holochila Schltr., 199

Protea

angolensis Welw.. 200

var. angolensis. 200

var. divaricata (Engl. \& Gilg) Beard. 200

baumii Engl. \& Gilg subsp. baumii. 200

chionamtha Engl. \& Gilg. 200

var. divaricata Engl. \& Gilg. 200

var. typica Engl. \& Gilg. 200

chrnsolepis Engl. \& Gilg. 200

gaguedi J.F.Gmel., 200)

haemantha Engl. \& Gilg. 200

lemairei De Wild. subsp. trichophylla (Engl. \& Gilg) Beard. 200

melliodora Engl. \& Gilg. 200

mursinifolia Engl. \& Gilg. 200

poggei Engl. subsp. haemantha Chisumpa \& Brummitt. 200

irichophylla Engl. \& Gilg. 200

welwitschii Engl., 200

Pycnosphaera

buchananii (Baker) N.E.Br., 196

trimera Gilg. 196

Quassia undulata (Guill. \& Perr.) D.Dietr., 201

Randia brachythammus K.Schum., 200

Rhus

commiphoroides Engl. \& Gilg. 191

polineura Engl. \& Gilg. 191

var. hylophila Engl. \& Gilg. 191

Rhunchosia

baumii Harms. 196

luteola (Hiern) K.Schum.. 196

moninensis Harms, 196

Rhynchospora

dolichostrla K.Schum., 195

holoschoenoides (Rich.) Herter. 195

Rorippa cryptantha (A.Rich.) Robyns \& Boutique. 193

Rytigynia orbicularis (K.Schum.) Robyns, 200

Salacia

bussei Loes.. 194

rehmanni Schinz. var baumii Loes. 194

Sapium suffruticosum Pax. 195

Satyrium

oliganthum Schltr. 199

paludosum Rchb.f. var. panibracteanum Schltr. 199

Sauvagesia africana (Baill.) Bamps, 198

Schizocarphus nervosus (Burch.) Van der Merwe. 196

Schizoglossum baumii Schltr. ex N.E.Br., 192

Scilla

baumiana Engl. \& Gilg, 196

eriospermoides Engl. \& Gilg. 196

lanceifolia ( Jacq.) Baker var. Iongiracemosa Engl. \& Gilg. 196

Sclerocroton oblongifolius (Müll.Arg.) Kruijt \& Roehers. 195

Searsia

kirkii (O)iv.) Moffet. 19

tenuinervis (Engl.) Moff̈et. 191

Sebaea

baumiana (Ciilg) Boutique, 196

teuszii (Schinz) P. Taylor. 196

Senecio

haumii (O.Hoffm. 193

cryphiactis (O.Hoffm.. 193 
Senecio (cont.)

limosus O.Hoffm., 193

relmatophilus O.Hoffm., 192

Sesamum

calycinum Welw. subsp, repens (Engl. \& Gilg) Seidenst., 199 repens Engl. \& Gilg. 199

Sesbania

coerulescens Harms, 196

microphylla Harms, 196

Sopubia

dregeana Benth. var. tenuifolia Engl. \& Gilg, 199

mannii Skan. 199

Sphaeranthus

flexuosus O.Hoffm. ex De Wild.. 193

humilis O.Hoffm., 193

Sphaerothvlax warmingiana Gilg, 199

Sphedamnocarpus

angolensis (A.Juss.) Planch. ex Oliv., 197

pulcherrimus Engl. \& Gilg, 197

Sporobolus

baumianus Pilg., 199

welwitschii Rendle, 199

Strobilanthopsis linifolia (T.Anderson ex C.B.Clarke) Milne-Redh.. 191

\section{Strychnos}

cocculoides Baker, 201

schumanniana Gilg. 201

Tacazzea

apiculata Oliv., 192

rosmarinifolia (Decne.) N.E.Br., 192

salicina Schltr., 192

verticillata K.Schum., 192

Tephrosia

cephalantha Welw. ex Baker, 196

coronilloides Welw. ex Baker, 196

hvpargirea Harms, 196

longana Harms, 196

Terminalia

baumii Engl. \& Gilg, 194

brachystemma Welw. ex Hicrn, 194

Thesium

leucanthum Gilg, 200

lycopodioides Gilg, 200

Tinnea

benguellensis Gürke, 197

coerulea Gürke var. coerulea. 197

fusco-luteola Gürke, 197

vestita Baker. 197
Tinospora caffra (Miers) Troupin, 198

Trachyandra arvensis (Schinz) Oberm., 192

Trichodesma baumii Gürke, 193

Trochomeria baumiana Gilg, 194

Tryphostemma haumii Harms, 199

Tulbaghia

aequinoctialis Welw. ex Baker subsp. monantha (Engl. \& Gilg) R.B.Burb., 191

monantha Engl. \& Gilg. 191

Tylophora caffra Meisn., 192

Utricularia

angolensis Kamienski, 197

baumii Kamienski, 197

spiralis $\mathrm{Sm} ., 197$

subulata L.. 197

Vangueria

lasioclados K.Schum., 200

proschii Briq., 200

Vausagesia bellidifolia Engl. \& Gilg, 198

Vernonia

haumii O.Hoffm., 193

brideliifolia O.Hoffm., 193

gerberiformis Oliv. \& Hiern subsp. macrocyanus (O.Hoffm.) C.Jeffrey, 193

limosa O.Hoffm., 193

mesogramme O.Hoffm., 193

primulina O.Hoffm., 193

pvgmaea (O.Hoffm., 193

subplumosa O.Hotfm., 193

temnolepis O.Hoffm., 193

Wahlenbergia

banksiana $\mathrm{A} . \mathrm{DC} ., 194$

cyanea Engl. \& Gilg, 194

denticulata (Burch.) A.DC., 194

leucamtha Engl. \& Gilg. 194

undulata (L.f.) A.DC., 194

Wulfhorstia ekehergioides Harms, 198

Xylotheca longipes (Gilg) Gilg. 196

Xvris

haumii L.A.Nilsson, 201

congensis Büttner, 201

Xysmalobium undulatum (L.) W.T.Aiton, 192

Zantedeschia

albomaculata (Hook.) Baill. subsp. albomaculata, 192

chloroleuca Engl. \& Gilg, 192

\section{INDEX OF BALM COLLECTION NUMBERS CITED AND TAXON UNDER WHICH THEY ARE CITED IN THIS PAPER}

32 Duosperma quadrangulare (Klotzsch) Brummitt

33 Euclea divinorum Hiern

43 Lobelia angolensis Engl. \& Diels ex Diels

47 Nidorella resedifolia $D C$. subsp. resedifolia

50 Nicolasia felicioides S.Moore

53 Combretum aureonitens Engl. \& Gilg

54, 56 Combretum collinum Fresen.

64 Dichapetalum cymosum (Hook.) Engl.

67 Artemisiopsis villosa (O. Hoffm.) Schweick

68 Senecio cryphiactis $O$.Hoffm.

88 Entandrophragma ekebergioides (Harms) Sprague

97 Ludwigia senegalensis (DC.) J. Troch.

98 Bergia pentheriana Keissl.

110 Bergia spathulata Schinz

112 Elytrophorus globularis Hack.

113 Combretum aureonitens Engl. \& Gilg

114 Ochna pulchra Hook.

117,119 Combretum platypetalum Welw. ex M.A.Lawson subsp. baumii (Engl. \& Gilg) Exell

120 Ottelia ulvifolia (Planch.) Walp.

121 Baphia massaiensis Taub. subsp. obovata (Schinz) Brummitt var. comifolia (Harms) Soladove

122 Scilla baumiana Engl. \& Gilg

122a Jatropha baumii Par

126 Grangea anthemoides $O$. Hoffm

127 Combretum molle $R$ Br. ex $G$.Don

129 Rorippa cryptantha (A. Rich.) Robvns \& Boutique

133 Physostigma mesoponticum Tauh.

134 Vernonia gerberiformis Oliv: \& Hiern subsp. macrocyanus $(O \mathrm{Hoffm}$.) C.Jeffrey
137 Leptactina pretrophylax K.Schum

144 Monopsis zeyheri (Sond) Thulin

145 Ochna pulchra Hook.

150 Asparagus baumii Engl \& Gilg

151 Ipomoea crassipes Hook.

155 Combretum molle R.Br. ex G.Don

172 Ochna pygmaea Hiern

174 Cyphostemma fugosioides (Gilg) Desc ex Wild \& $R$ B Drumm

184 Sphaeranthus flexuosus (O. Hoffm ex De Wild.

187 Erigeron baumii O.Hoffm

189 Grewia suffruticosa $\kappa$ Schum

191 Euclea crispa (Thunh.) Gürke

197 Melanthera triternata (Klaut) Wild

203a Ledebouria revoluta (L.f.) Jessop

204 Nesaca baumii Koehne

210 Fadogia chrysantha $K$.S.hum

211 Gnidia baumiana Gilg

215 Terminalia brachystemma Welw: ex Hiern

217 Phragmanthera baumii (Engl \& Gilg) Polhill \& Wien.

218 Thesium leucanthum Gilg

220 ()chna pyemaea Hiern

223 Erythrina baumii Harms

225 Buchnera baumii Engl. \& Gilg

226 Helichrysum congolanum Schltr \& OHotfm

232. 232a. 232b Combretum platypetalum Helw: ex M.A.Lawson subsp baumii (Engl \& Gilg) Erell

235 Vemonia pygmaca $0 . H o f f m$.

244 Polygala nambalensis Gürke

245 Tacazeca rosmarinifolia (Decne, $\triangle E B$ Br

252 Aeschynomene bracteosa Welw: ex Boker 
254 Indigofera nambalensis Harms

255 Vernonia limosa O.Hoffm.

256 Commelina milne-redheadii Faden

257 Sclerocroton oblongifolius (Müll.Arg.) Krnijt \& Roebers

261 Eulophia gonychila $S c h l t r$.

263 Hydrostachys triaxialis Engl. \& Gilg

270 Cyphostemma violaceo-glandulosum (Gilg) Desc.

272 Protea gaguedi J.F.Gmel.

273 Ammocharis baumii (Harms) Milne-Redh. \& Schweick.

275 Aloe zebrina Baker

276 Tacazzea apiculata Oliv.

276a Cyphostemma violaceo-glandulosum (Gilg) Desc.

278 Cissus jatrophoides (Welw: ex Baker) Planch.

283 Plectranthus baumii Gïrke

287 Polygala kalaxariensis Schinz

289 Grew ia avellana Hiern

290) Strychnos cocculoides Baker

301 Polygala arenicola Gürke

307 Utricularia spiralis $S m$

311,311 a Cyperus chrysocephalus (K. Schum.) Kük.

315a Polygala paludicola Gürke

316 Sebaea teuszii (Schinz) P.Tavlor

318 Pasaccardoa baumii O. Hoffm.

325 Nymphaca sulphurea Gilg

326 Brachycorythis angolensis (Schltr.) Schltr

332 Xysmalobium undulatum (L.) W.T.Aiton

333 Xyris congensis Bültner

341 Eulophia trilamellata De Wild.

342 Conyza welwitschii (S.Moore) Wild

349 Dipcadi vaginatum Baker

351 a Tulbaghia aequinoctialis Welw: ex Baker subsp. monantha (Engl \& Gilg) R.B.Burh.

353 Grewia falcistipula K.Schum.

354 Crassocephalum uvens (Hiern) S. Moore

355 Rhynchospora holoschoenoides (Rich.) Herter

357 Eulophia tricristata Schltr.

358 Conyza limosa ().Hoffm

361 Cyphostemma chloroleucum (Welw. ex Baker) Desc. ex Wild \& R.B.Drumm.

367 Eulophia coeloglossa Schltr.

369 Eulophia rolfeana Kraenzl.

373, 373a Polygala paludicola Gürke

374 Phaius occidentalis $S$ chltr:

375 Protea baumii Engl. \& Gilg subsp. baumii

377 Eulophia corymbosa Schltr.

381 Brachycorythis rhodostachys (Schltr) Summerh.

382 Eulophia calantha $S$ chltr.

383 Satyrium oliganthum $S$ chltr

384 Eulophia arenicola $S$ chltr.

388 Xylotheca longipes (Gilg) Gilg

389 Gardenia brachythamnus (K.Schum.) Launert

391 Basananthe baumii (Harms) W.J.de Wilde

398 Zantedeschia albomaculata (Hook.) Baill. subsp. albomaculata

402 Asparagus altiscandens Engl. \& Gilg

$4(13$ Cycnium tubulosum (L.f.) Engl. subsp. tubulosum

410 Schizocarphus nervosus (Burch.) Van der Merwe

412 Trachyandra arvensis ( $S$ chinz) Oherm

413 Aspidoglossum masaicum (N.E.Br.) Kupicha

420) (Grew ia schinzii $K S$ schum

421 Wahlenhergia banksiana A.DC.

$\$ 25$ Hahenaria huillensis $R$ chhb.t.

428 Baikiaca plurijuga Harms

$\$ 33$ Conyza limosa O Hoffm

445 Trechomeria baumiana Gilg

446 Vangueria proschii Briq.

447 L.edebouria revoluta (I.f.) Jessop)

454 Ipomoea crassipes Hook.

457 Cryptolepis productia N.E.Br

459 Dipcadi viride (L.) Moench

461 Anthericum liliagastrum Eingl \& Gilg

471 Entada arenaria Schinz subsp. arenaria

474 Inula limosa O.Hoffom

475 Sopubia mannii Skan

477 Wahlenbergia undulata (1.f.). . . DC

482 Fadogia chlorantha $K$ S chum var. chlorantha

483 Crotalaria sericifolia Horm

487 Bulbine abyssinica A. Rich.

491 Fadogia chlorantha $K$ Schum. var. thamnus $\left(K S_{\text {c }}\right.$.hum , Verde

496 Eulophia malangana (Rechb.f) Summerh
497 Indigofera baumiana Harms

499 Dipcadi longifolium (Lindl.) Baker

505 Eulophia adenoglossa (Lindl.) Rchb.f.

509 Ampelocissus brunneo-rubra Gilg

511 Sporobolus welwitschii Rendle

512 Ozoroa longipes (Engl. \& Gilg) R.Fern. \& A.Fern

514 Hermannia eenii Baker f.

515 Lippia baumii Gürke var. baumii

517 Chlorophytum psammophilum Engl. \& Gilg

519 Agelanthus terminaliae (Engl. \& Gilg) Polhill \& Wiens

522 Pavetta arenicola K. Schum.

523 Copaifera baumiana Harms

526 Tylophora caffra Meisn.

530 Anthericum xylorrhizum Engl. \& Gilg

535 Grewia avellana Hiern

540 Tinospora caffra (Miers) Troupin

544 Plectranthus betonicifolius Baker

550 Ochna cinnabarina Engl. \& Gilg

554 Polygala schinziana Chodat

561 Habenaria verdickii (De Wild.) Schlir.

563 Tephrosia cephalantha Welw. ex Baker

565 Gymnosporia senegalensis (Lam.) Loes.

569 Sesbania microphylla Harms

578 Habenaria holothrix Schlir.

581 Rytigynia orticularis (K. Schum.) Robyns

588 Sphedamnocarpus angolensis (A.Juss.) Planch. ex Oliv:

595 Polygala rivularis Gürke

598 Habenaria holubii Rolfe

603 Habenaria macroplectron Schlir

604 Neurotheca congolana De Wild. \& T.Durand

610 Vernonia brideliifolia $O$.Hoffm.

611 Chlorophytum colubrinum (Baker) Engl.

612 Tephrosia coronilloides Welw: ex Baker

614 Brachycorythis angolensis (Schltr.) Schltr.

616 Vernonia mesogramme $O$. Hoffm.

619 Burmannia madagascariensis Mart.

620 Sauvagesia africana (Baill.) Bamps

624 Vernonia limosa O. Hoffm

629 Brachycorythis thodostachys (Schltr.) Summerh

630 Inula limosa $O$. Hoffm

631 Eulophia calantha Schltr.

632 Gladiolus benguellensis Baker

638. 638a Searsia kirkii (Oliv.) Moffet

644 Platostoma strictum (Hiern) A.J.Paton

651 Gladiolus magnificus (Harms) Goldblatt

653 Protea welwitschii Engl.

657 Nymphaea sulphurea Gilg

659 Neopentanisia annua (K.Schum.) Verdc.

660 Sesamum calycinum Welw. subsp. repens (Engl. \& Gilg) Seidenst.

661 Clerodendrum baumii Gürke

662 Ozoroa stenophylla (Engl. \& Gilg) R.Fern. \& A.Fern.

664 Ozoroa xylophylla (Engl. \& Gilg) R.Fern \& A.Fern

669 Landolphia camptoloba (K.Schum.) Pichon

670) Crotalaria densicephala Welw. ex Baker

674 Quassia undulata (Guill. \& Perr.) D.Dierr.

691 Utricularia subulata $L$.

692 Sehaea baumiana (Gilg) Boutique

695 Tinnea vestita Baker

697 Tinnea benguellensis Gürke

698 Aloe nuttii Baker

699 Inula paludosa $O$. Hoffm

699a Asclepias baumii Schltr.

702 Sebaea teuszii (Schinz) P.Tavlor

703 Vernonia subplumosa OHoffm.

704 Polygala robusta Gürke

707 Emilia baumii (O.Hoffm) S Meore

$7(1)$ Protea poggei Engl. subsp. haemantha Chisumpa \& Brummint

710 Erlangea linearifolia (O.Hoffm.) S. Moore

712 Phyllocosmus lemaireanus (De Wild \& T.Durand) T.Durand \& H.Durand

713 Leptactina prostrata $K$ Schum

716 Aneilema plagiocapsa $K$.Schum

722 Combretum psidioides Welw. subsp. dinteri (Schinz) Exell

726 Burmannia madagascariensis Mart.

729 Limnophila dasyantha (Engl \& Gilg) Skan

73I Bulbine abyssinica 4 Rich

732 Julhemardia paniculata (Benth.) Troupin

734 Buchnera strictissima Engl \& Gilg

737 Acollanthus engleri Briq. 
739 Chironia baumiana Gilg

740) Crotalaria angulicaulis Harms

741 Crotalaria psammophila Harms

744 Searsia tenuinervis (Engl.) Moffer

745 Indigofera griseoides Harms

750 Limnophila ceratophylloides (Hiern) Skan

752 Pycnosphaera buchananii (Baker) N.E.Br

760 Pavonia senegalensis (Cav.) Leistner

764 Rhynchosia baumii Harms

772 Ottelia muricata (C.H.Wright) Dandv

779 Lepidagathis macrochila Lindau

780 Diospyros batocana Hiern

782 Sesbania coerulescens Harms

784 Erlangea linearifolia (O.Hoffm.) S. Moore

785 Baumia angolensis Engl. \& Gilg

787 Crotalaria subsessilis Harms

789 Plectranthus guerkei Briq.

792 Diospyros virgata (Gürke) Brenan

801 Anisopappus chinensis Hook. \& Arn. subsp. chinensis var. chinensis

804 Dicoma anomala Sond. subsp. anomala

805 Pavetta paupercula K.Schum.

808 Anisophyllea quangensis Engl. ex Henriq.

810 Aeschynomene baumii Harms

813, 813a Ochna arenaria De Wild. \& T.Durand

814 Commelina welwitschii C.B.Clarke

815 Polygala baumii Gürke

816 Polycarpaea inaequalifolia Engl. \& Gilg

817 Polygala nambalensis Gürke

819 Indigofera baumiana Harms

828 Gnidia pleurocephala Gilg

829 Crotalaria leptoclada Harms var. leptoclada

830 Strobilanthopsis linifolia (T.Anderson ex C.B.Clarke) Milne-Redh.

834 Diplolophium zambesianum Hiern

836 Crotalaria baumii Harms

837 Wahlenbergia denticulata (Burch.) A.DC.

838 Sebaea teuszii (Schinz) P. Taylor

839 Polystachya dendrobiiflora $R$ chb.f.

844 Trichodesma baumii Gürke

847 Bidens flabellata $O$. Hoffm.

848 Adenodolichos baumii Harms

857 Tinnea coerulea Gürke var. coerulea

858 Ottelia ulvifolia (Planch.) Walp.

863 Protea angolensis We/w. var. angolensis

866 Aeollanthus engleri Briq.
869 Harveya andongensis Hiern

871 Polygala nambalensis Giirke

872 Adenodolichos rhomboideus (O. Hoffm.) Harms var. rhomboideus

875 Buchnera prorepens Engl. \& Gilg

877 Anisopappus chinensis Hook. \& Arn. subsp. chinensis var. chinensis

879 Thesium lycopodioides Gilg

881 Gladiolus pallidus Baker

883 Bidens baumii (O.Hoffm.) Sherff

888 Monotes dasyanthus Gilg

891 Aloe metallica Engl. \& Gilg

892 Ozoroa argyrochrysea (Engl. \& Gilg) R.Fern. \& A.Fern

894 Cephalaria retrosetosa Engl. \& Gilg

898 Gladiolus pallidus Baker

899 Calanda rubricaulis K.Schum. var. rubricaulis

903 Protea welwitschii Engl.

904 Ledermanniella warmingiana (Gilg) C.Cusset

906 Anagallis filifolia Engl. \& Gilg

907 Emilia limosa $(O$. Hoffm.) C. Jeffrey

909 Hypericum lalandii Choisy

910 Benguellia lanceolata (Gürke) G. Tavlor

9II Polygala rivularis Gïrke

912 Faurea intermedia Engl. \& Gilg

915 Floscopa flavida C.B.Clarke

918 Protea lemairei De Wild. subsp. trichophylla (Engl. \& Gilg) Beard

920 Protea angolensis Welw. var. divaricata (Engl. \& Gilg) Beard

923 Dopatrium stachytarphetoides Engl. \& Gilg

926 Dicoma antunesii $O$. Hoffm

929, 929a Vernonia temnolepis (O.Hoffm.

933 Dicoma anomala Sond. subsp. anomala

938 Kalanchoe brachyloba Welw: ex Britten

941 Ozoroa argyrochrysea (Engl. \& Gilg) R Fern \& A.Fern.

942 Duosperma sessilifolium (Lindau) Brummitt

945 Diospyros kirkii Hiern

947 Combretum molle R. Br. ex G.Don

948 Pavetta stipulopallium K.Schum.

962 Ottelia kunenensis (Gürke) Dandw

963 Commelina subulata Roth

966 p.p. quoad fructus Kirkia acuminata Oliv.

981 Combretum collinum Fresen.

982 Combretum aureonitens Engl \& Gilg

983, 986 Combretum collinum Fresen.

999 Boscia welw itschii Gilg

1005a Rhynchosia luteola (Hiern) K.Schum.

APPENDIX 1.- Main geographical names cited in Warburg (1903) as collecting localities or shown on his map [included in book]. with corresponding actual name and geographical co-ordinates

\begin{tabular}{|c|c|c|c|c|c|}
\hline $\begin{array}{l}\text { Original name in } \\
\text { Warburg (1903) }\end{array}$ & $\begin{array}{l}\text { Portuguese names on maps } \\
\text { consulted }\end{array}$ & $\mathrm{S}$ & $\mathrm{E}$ & Source* & Notes \\
\hline Alixandre & $\begin{array}{l}\text { Fazenda do Alexandre; } \\
\text { Alexandre }\end{array}$ & $15.52(15.48)$ & $12.09(12.06)$ & $\begin{array}{l}\text { [4] Sheet no. 3: [5] } \\
\text { Sheet no. I }\end{array}$ & \\
\hline Andara & Andara & 18.03 & 21.25 & [2] & \\
\hline Bindi & Lagoa Bindi & 17.05 & 18.40 & [2] & \\
\hline Buraco & Buraco & $15.35(15.37)$ & $12.12(12.11)$ & [2]; [5] Sheet no. 1 & \\
\hline Cabindere & Cavindera; Cabindele & $15.50(15.52)$ & $17.36(17.35)$ & [2]: [3] Sheet no. 385 & \\
\hline Cacimba & Cacimba & 15.32 & 12.02 & [5] Sheet no. I & \\
\hline Camelungu & Lagoa Camelungu & 16.58 & 18.44 & {$[2]$} & \\
\hline Canongofiepfanne & Lagoa Canangossi & 16.01 & 15.24 & [5] Sheet no. 2 & $\begin{array}{l}\text { This lake has apparently disap- } \\
\text { peared as it is not shown on recent } \\
\text { maps examined. Baum's itinerary } \\
\text { follows paths marked on map [5]. }\end{array}$ \\
\hline Carvalhao & Lagoa do Carvalhão & 15.45 & 12.06 & [3] Sheet no. 374 & \\
\hline Chibia & $\begin{array}{l}\text { Chibia; Vila Joào de } \\
\text { Almeida }\end{array}$ & 15.11 & 13.42 & $\begin{array}{l}\text { [3] Sheet no. 356: [2]: } \\
\text { [5] }\end{array}$ & \\
\hline Chihinde & Chindinde & 16.32 & 14.05 & [3] Sheet no. 421 & \\
\hline
\end{tabular}

* Publications and maps consulted:

[1] Warburg, O. 1903. Kunene-Sambesi-Expedition. H. Baum. Kolonial-Wintschaftliches Komitec, Berlin.

[2] Carta da Colónia de Angola (Esboço). Ministério das Colónias, Junta das Missỏes Geográficas e de investigaçỏes Coloniais. 1935 Esc. 1: 1.500 .000 .

[3] Carta de Angola, Ministério do Ultramar, Junta das Missöes Geográficas e de investigaçòes Coloniais. Esc. 1: 1(0).(1)(X).

[4] Carta da Colónia de Angola, Ministério das Colónias, Junta das Missōes (jeográficas é de investigaçóes Coloniais. 1947. Esc. 1: 2.(X)().(K)).

[5] Carta do Sul de Angola, Ministério das Colónias, Comissão de Cartografia. 1929-1932. Esc. 1: 500.(000).

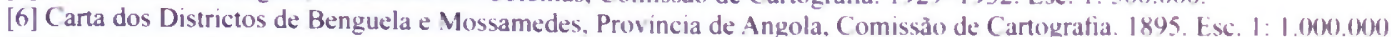

[7] Carta de Angola, Junta de Investigaçòes do Lltramar. 1973. Esc. 1: 2 (1)(0) (0)(0).

[8] Angola Gazetteer. USGS. 
APPENDIX 1.-Main geographical names cited in Warburg (1903) as collecting localities or shown on map included in book, with corresponding actual name and geographical co-ordinates (cont.)

\begin{tabular}{|c|c|c|c|c|c|}
\hline $\begin{array}{l}\text { Original name in } \\
\text { Warburg (1903) }\end{array}$ & $\begin{array}{l}\text { Portuguese names on maps } \\
\text { consulted }\end{array}$ & $\mathrm{S}$ & $E$ & Source* & Notes \\
\hline $\begin{array}{l}\text { Chijija (Chỹÿa on } \\
\text { map) }\end{array}$ & Chicungo & 15.47 & 18.38 & [3] Sheet no. 387 & $\begin{array}{l}\text { Location in }[1] \text { coincides with } \\
\text { Chicungo. }\end{array}$ \\
\hline Chitanda & R. Chitanda: R. Calonga & & & {$[2] ;[3]$} & $\begin{array}{l}\text { As Chitanda R. in [2] and Calonga } \\
R \text {. in [3]. }\end{array}$ \\
\hline Cicalele-Calilile & Calalele & 15.08 & 20.18 & {$[2]$} & \\
\hline Ediva & Ediva & 16.20 & 14.15 & $\begin{array}{l}\text { [3] Sheet no. 399; [5] } \\
\text { Sheet no. 2: [2] }\end{array}$ & \\
\hline Fort Tschimbemba & Chibemba; Chivemba & 15.45 & 14.05 & $\begin{array}{l}\text { [3] Sheet no. } 378 ;[2] \\
\text { [5] Sheet no. 2: }[4] \\
\text { Sheet no. } 3\end{array}$ & \\
\hline Goudkopje & & 15.19 & 15.55 & [3] Sheet no. 360 & $\begin{array}{l}\text { Exact location may be near these } \\
\text { co-ordinates. }\end{array}$ \\
\hline Habungu & & & & {$[7]$} & Appears to be Chissombo R. \\
\hline $\begin{array}{l}\text { Hadjon Longa ( } 3 \text { dots } \\
N \text { on map) }\end{array}$ & & 16.06 & 19.07 & [3] Sheet no. 409 & $\begin{array}{l}\text { There is no indication of settlement } \\
\text { in this location. Co-ordinates given } \\
\text { here represent a medium location } \\
\text { between Palai R. (Palé) and Cuma } \\
\text { R. (Mocuma). }\end{array}$ \\
\hline $\begin{array}{l}\text { Hadjon Longa ( } 3 \text { dots } \\
\mathrm{S} \text { on map) }\end{array}$ & & 16.15 & 19.07 & [3] Sheet no. 409 & $\begin{array}{l}\text { Co-ordinates given here represent a } \\
\text { medium location between Cuma R. } \\
\text { (Mocuma) and Cacito R. (Cafito). } \\
\text { on left bank of Cuito R. }\end{array}$ \\
\hline Hai & Hac; Haii & 15.27 & 13.50 & $\begin{array}{l}\text { [5] Sheet no. 2; [3] } \\
\text { Sheet no. 356; [2] }\end{array}$ & \\
\hline Hartebeestpfanne & & 16.04 & 15.27 & $\begin{array}{l}\text { [5] Sheet no. 2: [3] } \\
\text { Sheet no. } 401\end{array}$ & $\begin{array}{l}\text { These may be three lakes that have } \\
\text { been mapped with the name Etalas } \\
\text { Camunhandi. Co-ordinates are } \\
\text { those given on map [5]. }\end{array}$ \\
\hline Humbe & Humbe & 16.41 & 14.54 & $\begin{array}{l}\text { [3] Sheet no. 422; [4] } \\
\text { Sheet no. } 3 ;[2] ;[5]\end{array}$ & \\
\hline Jai & Jai & 14.42 & 17.38 & [3] Sheet no. 344 & \\
\hline Kafa & Cafu; Cáfu & 16.27 & 15.07 & $\begin{array}{l}\text { [3] Sheet no. } 401 ;[5] \\
\text { Sheet no. } 2 ;[4] \text { Sheet } \\
\text { no. } 3 ;[2]\end{array}$ & $\begin{array}{l}\text { Co-ordinates correspond to a loca- } \\
\text { tion on left bank and not to place } \\
\text { named Cafu ( } \mathrm{C} \text { afú) on map. }\end{array}$ \\
\hline Kahama & Cahama & 15.17 & 14.18 & $\begin{array}{l}\text { [3] Sheet no. } 399 ;[2] ; \\
\text { [5] Sheet no. } 2 ;[4] \\
\text { Sheet no. } 3\end{array}$ & \\
\hline Kakele & Calima & 15.14 & 16.02 & [3] Sheet no. 361 & \\
\hline Kakulovar & R. Caculuvar & & & [7] & \\
\hline Kalolo & Calola & 17.08 & 18.14 & [3] Sheet no. 452 & \\
\hline Kamba & $\begin{array}{l}\text { Pecuária da Camba: } \\
\text { Embala da Camba: Camba }\end{array}$ & 16.16 & 15.15 & $\begin{array}{l}{[3] \text { Sheet no. } 401 ;[5]} \\
\text { Sheet no. } 2 ;[2]:[4] \\
\text { Sheet no. } 3\end{array}$ & \\
\hline Kampuluve & R. Cumpulua & & & {$[7]$} & \\
\hline Kanjundu & Caiundo & 15.42 & 17.27 & $\begin{array}{l}\text { [3] Sheet no. } 384:[5] \\
\text { Sheet no. } 2:[2] ;[4] \\
\text { Sheet no. } 3:[6]\end{array}$ & \\
\hline Kapengombe & Capangombe & $15.07(15.06)$ & $13.07(13.08)$ & $\begin{array}{l}\text { [5] Sheet no. 1: [2]: [3] } \\
\text { Sheet no. } 355\end{array}$ & \\
\hline Kassinga & Cassinga & 15.07 & 16.05 & $\begin{array}{l}\text { [3] Sheet no. 361; [2]: } \\
\text { [5] Sheet no. } 2 ;[4] \\
\text { Sheet no. } 3\end{array}$ & \\
\hline $\begin{array}{l}\text { Katholische Mission } \\
\text { (Kassinga) }\end{array}$ & $\begin{array}{l}\text { Missão Católica de Cas- } \\
\text { singa }\end{array}$ & 15.06 & 16.05 & [6] Sheet no. 3 & $\begin{array}{l}\text { On this map, the mission appears } \\
\text { mapped south of Cassinga. not } \\
\text { north as it appears on [1]. }\end{array}$ \\
\hline Katumba & Catomba & 15.20 & 13.37 & [3] Sheat no. 356 & \\
\hline Kavumba & & 15.31 & 19.15 & [3] Sheet no. 367 & $\begin{array}{l}\text { No village could be located. Co- } \\
\text { ordinates correspond to a location } \\
\text { near source of Chiova R. (Vijowa). }\end{array}$ \\
\hline Kaw anga & Cabanga & 17.04 & 18.10 & [2] & \\
\hline Kingu & Quengue"? & 16.36 & 15.07 & [6] Sheet no. 3 & \\
\hline
\end{tabular}

* Publications and maps consulted:

[1] Warburg, O. 19(1)3. Kunene-Sambesi-Expedition. H. Baum. Kolonial-Wirtschaftliches Komitee. Berlin.

[2] Carta da Colónia de Angola (Esboço). Ministério das Colónias. Junta das Missỏes Geográficas e de investigaçòes Coloniais. 1935. Esc. 1: $1.5(70.00(0)$

[3] Carta de Angola. Ministério do Ultramar. Junta das Missies Geograficas e de investigaçỏes Coloniais. Esc. 1: 100.000.

[4] Carta da Colónia de Angola, Ministério das Colonias. Junta das Missôes Geográficas e de investigações Coloniais. 1947. Esc. 1: 2.(000).000.

[5] Carta do Sul de Angola. Ministério das Colónias. Comissảo de Cartografia. 1929-1932. Esc. 1: 500.(0)0

[6] Carta dos Districtos de Benguela e Mossamedes, Provincia de Angola, Comissão de (anografia. 1895. Esc. 1: 1 . (M0).(00).

[7] Carta de Angola. Junta de Investigaçèes do Ultramar. 1973. Esc. 1: 2 (4)(0) 0(0).

[8] Angola Gazetterer. USGS. 
APPENDIX 1.- Main geographical names cited in Warburg (1903) as collecting localities or shown on map included in book, with corresponding actual name and geographical co-ordinates (cont.)

\begin{tabular}{|c|c|c|c|c|c|}
\hline $\begin{array}{l}\text { Original name in } \\
\text { Warburg (1903) }\end{array}$ & $\begin{array}{l}\text { Portuguese names on maps } \\
\text { resources }\end{array}$ & $\mathrm{S}$ & $\mathrm{E}$ & Source* & Notes \\
\hline Kiteve & Quiteve; Chiteve & 16.01 & 15.11 & $\begin{array}{l}\text { [3] Sheet no. } 401 ;[2] ; \\
\text { [5] Sheet no. } 2 ;[4] \\
\text { Sheet no. } 3\end{array}$ & \\
\hline Kohi & $\begin{array}{l}\text { Vila da Ponte; Vila Artur } \\
\text { de Paiva }\end{array}$ & 14.28 & 16.18 & $\begin{array}{l}\text { [3] Sheet no. } 321 ;[2] \text {; } \\
\text { [5] Sheet no. } 2 ;[4] \\
\text { Sheet no. } 3\end{array}$ & $\begin{array}{l}\text { As Vila da Ponte on [2] and Vila } \\
\text { Artur de Paiva on [3] Sheet no. } \\
321 \text {. }\end{array}$ \\
\hline Konjongo & Caiongo & 15.33 & 19.50 & {$[2]$} & \\
\hline Kubango & R. Cubango & & & [7] & \\
\hline Kuebe & R. Cuebe & & & [7] & \\
\hline Kueio & R. Cueio & & & [7] & \\
\hline Kuelleis & R. Colui? & & & {$[7]$} & \\
\hline Kuimara & & 17.36 & 18.37 & $\begin{array}{l}\text { [3] Sheet no. } 463 ;[2] ; \\
\text { [5] Sheet no. } 2 ;[4] \\
\text { Sheat no. } 3\end{array}$ & $\begin{array}{l}\text { This name could not be found but it } \\
\text { appears to correspond to Cuangar, } \\
\text { since Habungu R. [1] appears to be } \\
\text { Bungo R. or Puca R. [2] and Chis- } \\
\text { sombo [7]. }\end{array}$ \\
\hline Kuito & R. Cuito & & & [7] & \\
\hline Kulei & R. Cuelei & & & [7] & \\
\hline Kunene & R. Cunene & & & [7] & \\
\hline Kusisi & R. Cuzizi & & & {$[7]$} & \\
\hline Kutsi & R. Cuchi & & & [7] & \\
\hline Lazingua & R. Luassingua & & & {$[7]$} & \\
\hline $\begin{array}{l}\text { Lihurna ("Destroyed } \\
\text { by Kuangaris") }\end{array}$ & & 15.12 & 19.23 & [3] Sheet no. 367 & $\begin{array}{l}\text { Co-ordinates are for Sampuloto, } \\
\text { which corresponds to the point } \\
\text { given on [1]. }\end{array}$ \\
\hline $\begin{array}{l}\text { Likise (Agent } \\
\text { C.D.M.) }\end{array}$ & & 15.29 & 19.38 & [3] Sheet no. 368 & $\begin{array}{l}\text { Co-ordinates refer to an unnamed } \\
\text { native village which is located in } \\
\text { same place as this name on [1]. }\end{array}$ \\
\hline Lupembe & Lupembe & 16.39 & 14.52 & [5] Sheet no. 2 & \\
\hline Makopi & Macope & 15.54 & 14.09 & [6] Sheet no. 3 & \\
\hline Makuju & $\begin{array}{l}\text { Macujo; Lagoa Macon- } \\
\text { guele }\end{array}$ & $16.26(16.57)$ & $18.47(18.50)$ & [2]: [3] Sheet no. 430 & $\begin{array}{l}\text { Even though the latitude of this } \\
\text { lake is different from that on [1], } \\
\text { there are no other lakes mapped in } \\
\text { the area. }\end{array}$ \\
\hline Manonge & Serpa Pinto; Menongue & 14.39 & 17.41 & $\begin{array}{l}\text { [3] Sheet no. 344: [2]; } \\
\text { [4] Sheet no. } 3\end{array}$ & \\
\hline Manonge 1 & & 14.42 & 17.39 & [3] Sheet no. 344 & $\begin{array}{l}\text { Near Tucuve R. On [3] Sheet no. } \\
344 \text { corresponds to Muanangombe. }\end{array}$ \\
\hline Maschonge & R. Massongue & & & {$[7]$} & \\
\hline Massaca & Massaca & 15.35 & 17.24 & [4] Sheet no. 3; [2] & \\
\hline Matonga & Matonga; Malonga & 15.30 & 20.17 & {$[2] ;[4]$ Sheet no. 6} & $\begin{array}{l}\text { This locality is east of Ngombe } R \text {., } \\
\text { whereas on [1] it appears west of } \\
\text { that river (Ongombe } R \text {.). Taking } \\
\text { into account the tracks mapped on } \\
\text { [2] which Baum most probably fol- } \\
\text { lowed, we suppose this is the same } \\
\text { Matonga. }\end{array}$ \\
\hline Matukua & Mutucua & 16.28 & 14.35 & [5] Sheet no. 2 & \\
\hline Menempremp & & 14.42 & 16.36 & [3] Sheet no. 342 & $\begin{array}{l}\text { There is a Mema Grande R., south } \\
\text { of Vulumba R., that is also a tribu- } \\
\text { tary of Cubango R. Co-ordinates } \\
\text { given here are those of the conflu- } \\
\text { ence of Mema Crande-Cubango } \\
\text { Rivers. }\end{array}$ \\
\hline Meschekke & Area de Muchequecheque & 15.36 & 13.47 & [3] Sheet no. 377 & $\begin{array}{l}\text { Co-ordinates of Tomba vil- } \\
\text { lage, which is located in } \\
\text { Muchequecheque area. }\end{array}$ \\
\hline Minjanja (Norte) & & 15.35 & 19.41 & [3] Sheet no. 389 & $\begin{array}{l}\text { Location of an unnamed village. } \\
\text { based on the point mapped on [1]. }\end{array}$ \\
\hline
\end{tabular}

\footnotetext{
* Publications and maps consulted:

[1] Warburg, O. 1903. Kunene-Sambesi-Expedition. H. Baum. Kolonial-Wirtschaftliches Komitee, Berlin.

[2] Carta da Colónia de Angola (Esboço), Ministério das Colónias, Junta das Missỏes Geográficas e de investigações Coloniais. 1935. Esc. I: 1.500 .000 .

[3] Carta de Angola, Ministério do Ultramar, Junta das Missões Geográficas e de investigaçoes Coloniais. Esc. 1: $100.0(0)$.

[4] Carta da Colónia de Angola, Ministerio das Colónias. Junta das Missỏes (jeográficas e de investigaçiés Coloniais. 1947. Esc. I: 2.(KK).(KK).

[5] Carta do Sul de Angola, Ministério das Colónias, Comissão de Cartografia. 1929-1932. Esc. 1: 5(x).(x) .

[6] Carta dos Districtos de Benguela e Mossamedes. Provincia de Angola, Comissão de Cartografia. 1895. Esc. 1: 1.000).000).

[7] Carta de Angola. Junta de Investigações do Ultramar. 1973. Esc. 1: 2 (0)00 (0)(0).

[8] Angola Gazetteer. USGS
} 
APPENDIX 1.- Main geographical names cited in Warburg (1903) as collecting localities or shown on map included in book, with corresponding actual name and geographical co-ordinates (cont.)

\begin{tabular}{|c|c|c|c|c|}
\hline $\begin{array}{l}\text { Original name in } \\
\text { Warburg }(1903)\end{array}$ & $\begin{array}{l}\text { Portuguese names on maps } \\
\text { consulted }\end{array}$ & $\mathrm{S}$ & $E$ & Source* \\
\hline Minjanja (Sul) & & 15.37 & 19.44 & [3] Sheet no. 389 \\
\hline Minnesera & & & & [7] \\
\hline Mitjelaga & Michelaga & 15.14 & 20.18 & {$[2]$} \\
\hline Mossamedes & $\begin{array}{l}\text { Moçâmedes; Mossamedes; } \\
\text { Mossâmedes }\end{array}$ & 15.12 & 12.09 & $\begin{array}{l}\text { [3] Sheet no. 353; [2 } \\
\text { [5]; [4] Sheet no. } 3\end{array}$ \\
\hline Mukuma & Mucuma & 15.00 & 13.34 & $\begin{array}{l}\text { [3] Sheet no. 356: [2 } \\
\text { [5] }\end{array}$ \\
\hline Mundongo & Indungo & 14.49 & 16.16 & $\begin{array}{l}\text { [3] Sheet no. 341: [2] } \\
\text { [4] Sheet no. 3; [5] } \\
\text { Sheet no. } 2\end{array}$ \\
\hline Nambali & R. Bale & & & {$[7]$} \\
\hline Napalanka & & 16.15 & 18.52 & [3] Sheet no. 408 \\
\hline Nevis & Neves & 15.07 & 12.54 & [5] Sheet no. 1; [2] \\
\hline Ondongi-Liwingi & Ondongi & 15.23 & 20.17 & [2] \\
\hline Onjimba & & 16.36 & 19.05 & [3] Sheet no. 431 \\
\hline
\end{tabular}

\section{Onschingue}

\begin{tabular}{|c|c|c|c|c|}
\hline Otjenjau & Otchinjau & 16.30 & 13.55 & $\begin{array}{l}\text { [3] Sheet no. 420; [2]; } \\
\text { [5] Sheet no. 2: [4] } \\
\text { Sheet no. } 3\end{array}$ \\
\hline Otjicutsie & Chicusse; Tchicusse & 16.22 & 14.27 & $\begin{array}{l}\text { [3] Sheet no. 399; [2]; } \\
\text { [5] Sheet no. 2: }[4] \\
\text { Sheet no. } 3\end{array}$ \\
\hline Palmfontein & $\begin{array}{l}\text { Oncócua: Ongogoa; Fonte } \\
\text { das Palmeiras; Uncocua }\end{array}$ & 16.39 & 13.25 & $\begin{array}{l}\text { [3] Sheet no. 419: [2]; } \\
\text { [5]: [4] Sheet no. } 3\end{array}$ \\
\hline Petri Girande & Pedra Grande & 15.00 & 12.36 & [5] Sheet no. 1; [2] \\
\hline Pokolo & Pocolo & 16.24 & 15.14 & $\begin{array}{l}\text { [3] Sheet no. 401; [5] } \\
\text { Sheet no. } 2\end{array}$ \\
\hline Port Alexandre & Porto Alexandre & 15.48 & 11.51 & $\begin{array}{l}\text { [3] Sheet no. 373; [2]; } \\
\text { [5] }\end{array}$ \\
\hline Quatiri & R. Cuatir & & & [7] \\
\hline Quiriri & R. Cuiriri & & & [7] \\
\hline Sakkemecho & & 14.42 & 18.39 & [3] Sheet no. 346 \\
\hline
\end{tabular}

Sambento do Sul

Subida

Tjimbanda

Tjipelongo

Tondiva

Tschiculecandi

Umpupe

Wolomber
São Bento do Sul

Subida

Chimbanda

Tchipelongo; Chipelongo

Tuandiva

Lagoa Chiculecondi

Ompupa: Umpupa

$\begin{array}{ll}15.48(15.49) & 12.06(12.06) \\ 15.22 & 12.08 \\ 14.59 & 20.18 \\ 16.29 & 14.37\end{array}$

16.33

16.53

16.16

14.39
14.44

18.46

13.29

16.31
Notes

Location of the Mucoboto village, based on the point mapped on [1].

Not listed in sources searched. it is situated at confluence of Longa and

Cuiriri Rivers [1].

There is no village with this name. Co-ordinates refer to an approximate location considering the course of the rivers mapped on [1]

Taking into account the course of the rivers on [1], these co-ordinates correspond to approximate location of an unnamed village.

Tributary of Cuito R., south of Campulua [1].

Co-ordinates are for Masseca, in the Micango area. This is based on the actual track [2] which Baum may have followed.

[2]; [5] Sheet no. 1

[5] Sheet no. 1

[2]

[3] Sheet no. 400: [4] Sheet no. 3: [2]; [5]

Sheet no. 2

[3] Sheet no. 422: [5]

Sheet no. 2

[2]

[3] Sheat no. 397: [2]: [5]

[3] Sheet no. 342
Co-ordinates refer to confluence of Vulumba and Cuhango Rivers.

\footnotetext{
* Publications and maps consulted:

[1] Warburg. O. 1903. Kunene-Sambesi-Expedition. H. Baum. Kolonial-Wirtschaftliches Komitee. Berlin.

[2] Carta da Colonia de Angola (Esboço). Ministério das Colónias. Junta das Missỏes Geográficas e de investigaçôes Coloniais, 1935 Esc. $1: 1.500 .000$

[3] Carta de Angola. Ministerio do Ultramar. Junta das Missiés Geograficas e de investigaçōes Coloniais. Esc. 1: 100.(100)

14] Carta da Colonia de Angola, Ministério das Colonias, Junta das Missōes Geográficas e de investigaç̃es Coloniais. 1947. Esc. 1: 2 .000 (ono.

[5] Carta do Sul de Angola. Ministério das Colonias. Comissão de Cartografia. 1929-1932. Esc. 1: $5($ (x).(000)

16] Carta dos Districtos de Benguela e Mossamedes, Provincia de Angola. Comissão de Cartografia. 1895. Esc. 1: 1.000).(0)(1).

[7] Carta de Angola, Junta de Investigacées do Ultramar. 1973. Esc. 1: 2 (f)( ) ()(M).

[8] Angola Gazetteer. USGS
} 